Published in final edited form as:

Nat Methods. 2020 February ; 17(2): 225-231. doi:10.1038/s41592-019-0676-4.

\title{
Nanoscale subcellular architecture revealed by multicolor 3D salvaged fluorescence imaging
}

\author{
Yongdeng Zhang ${ }^{1,11}$, Lena K. Schroeder ${ }^{1,11}$, Mark D. Lessard ${ }^{1}$, Phylicia Kidd ${ }^{1}$, Jeeyun \\ Chung ${ }^{1,2,3}$, Yuanbin Song ${ }^{4}$, Lorena Benedetti ${ }^{1,2,3}$, Yiming Li $^{5}$, Jonas Ries ${ }^{5}$, Jonathan B. \\ Grimm $^{6}$, Luke D. Lavis ${ }^{6}$, Pietro De Camilli ${ }^{1,2,3,7}$, James E. Rothman ${ }^{1,8}$, David Baddeley ${ }^{1,8,9}$, \\ Joerg Bewersdorf $1,7,8,10$,
}

${ }^{1}$ Department of Cell Biology, Yale School of Medicine, New Haven, Connecticut, USA ${ }^{2}$ Department of Neuroscience, Yale School of Medicine, New Haven, Connecticut, USA ${ }^{3}$ Howard Hughes Medical Institute, Yale School of Medicine, New Haven, Connecticut, USA ${ }^{4}$ Section of Hematology, Department of Internal Medicine, Yale School of Medicine, New Haven, Connecticut, USA ${ }^{5}$ Cell Biology and Biophysics Unit, European Molecular Biology Laboratory, Heidelberg, Germany ${ }^{6}$ Janelia Research Campus, Howard Hughes Medical Institute, Ashburn, Virginia, USA ${ }^{7}$ Kavli Institute for Neuroscience, Yale School of Medicine, New Haven, Connecticut, USA ${ }^{8}$ Nanobiology Institute, Yale University, West Haven, Connecticut, USA ${ }^{9}$ Auckland Bioengineering Institute, University of Auckland, Auckland, New Zealand ${ }^{10}$ Department of Biomedical Engineering, Yale University, New Haven, Connecticut, USA ${ }^{11}$ These authors contributed equally to this work

\section{Abstract}

Combining the molecular specificity of fluorescent probes with three-dimensional (3D) imaging at nanoscale resolution is critical for investigating the spatial organization and interactions of cellular organelles and protein complexes. We present a super-resolution light microscope that enables simultaneous multicolor imaging of mammalian cells at 5-10 nm single-molecule localization precision in 3D. We show its power for cell biology research with fluorescence images that resolve the highly convoluted Golgi apparatus and the close contacts between the endoplasmic reticulum and the plasma membrane, structures that have traditionally been the imaging realm of electron microscopy.

\footnotetext{
Users may view, print, copy, and download text and data-mine the content in such documents, for the purposes of academic research, subject always to the full Conditions of use:http://www.nature.com/authors/editorial_policies/license.html\#terms

*Corresponding author. joerg.bewersdorf@yale.edu (J.B.).

Author contributions

Y.Z. and J.B. conceived the idea. Y.Z. implemented the hardware. Y.Z., Y.L., J.R., and D.B. wrote the software. L.K.S., J.C., P.D.C., J.E.R., and J.B. designed biological experiments. L.K.S. developed sample preparation protocols. L.K.S. prepared and optimized microtubule, ER, mitochondria, Golgi, and ER-PM contact sites samples. Y.S. and L.B. provided the synaptonemal complex samples M.D.L. prepared and optimized the synaptonemal complex samples. P.K. prepared and optimized microtubule samples. Y.Z. imaged the samples and analyzed the images. J.B.G. and L.D.L. synthesized PA-JF646 and PA-JF669. Y.Z., L.K.S., and J.B. wrote the manuscript with input from all authors. All authors discussed the results and commented on the manuscript.

Competing interests

J.B. has financial interests in Bruker Corp. and Hamamatsu Photonics. J.B. is co-inventor of a U.S. patent application (US20170251191A1) related to the 4Pi-SMS system and image analysis used in this work. Y.Z. and J.B. have filed a U.S. patent application about the salvaged fluorescence multicolor imaging method described in this work.
} 


\section{Introduction}

While 'form follows function' is a well-established principle in architecture, resolving subcellular morphology to understand basic operating principles of a cell has been hampered by a lack of suitable imaging tools. Revealing the intricate inner workings of cells requires visualizing the interactions between proteins and organelles with molecular specificity at nanoscale resolution in three dimensions (3D). The diffraction-limited resolution of conventional light microscopy (about $250 \mathrm{~nm}$ ) stands in stark contrast to the structural dimensions of many organelles and complexes, such as the thickness of Golgi cisternae (about $50 \mathrm{~nm}$ each) ${ }^{1}$ and the diameter of tubules of the endoplasmic reticulum (ER) (about $80-100 \mathrm{~nm})^{2}$. Electron microscopy (EM), while providing sufficient resolution with ease, lacks many of the tools that offer molecular specificity in fluorescence light microscopy. Recently developed fluorescence super-resolution techniques have overcome the diffraction barrier and achieved impressive resolutions ${ }^{3,4}$. The ultimate goal, however - simultaneously resolving multiple targets of interest, for example the spatial relationship between two proteins in the context of a related organelle in 3D - is still very challenging and has constrained the impact of super-resolution microscopy in cell biology.

To address this challenge, we set out to develop a super-resolution instrument which can obtain high-quality images in three color channels, i.e. better than $10 \mathrm{~nm}$ localization precision in 3D, high molecular detection efficiency and negligible channel shift and crosstalk. Two previous inventions in the super-resolution field form the foundation of our development: (i) interferometric detection of fluorescence from individual emitters by two opposing objectives in a '4Pi' geometry with single-molecule switching (4Pi-SMS) has demonstrated an improvement in axial localization precision matching or surpassing the lateral values ${ }^{5-8}$. This imaging modality has also been shown to obtain multicolor data of biological structures close to the coverslip by sequential imaging ${ }^{8-10}$. However, multicolor imaging over the whole depth of a cell remains difficult as the channel registration becomes challenging when imaging deep in the samples due to the depth-dependent distortions ${ }^{11,12}$ and the refractive index heterogeneities within the specimens ${ }^{13}$ (Supplementary Note 1). (ii) Ratiometric color assignment can determine molecular identities based on the spectral information extracted from spectrally similar, simultaneously imaged fluorescent emitters ${ }^{14-19}$. This approach allows for the use of multiple far-red dyes, many of which have been shown to outperform the majority of dyes in other wavelength ranges with regards to the number of detected photons per switching event, on-off duty cycle and number of switching cycles ${ }^{18,20}$, and reduces the chromatic aberrations. Ratiometric color assignment has struggled so far, however, with obtaining high assignment efficiency without rejecting or falsely assigning large fractions of molecules, and doing so without substantially compromising localization precision. Combining interferometric 4Pi-SMS imaging with a refined ratiometric detection scheme which takes advantage of 'salvaged fluorescence' (SF), we show in this work imaging of mammalian cells at 5-10 nm localization precision in 3D in three colors simultaneously. 


\section{Results}

\section{Implementation of multicolor 4Pi-SMS using salvaged fluorescence}

Ratiometric single-molecule imaging assigns molecular identity by comparing the singlemolecule emitter signal levels detected in two or more spectral windows ${ }^{14-19}$. If emission spectra are known and the signal to noise ratio is sufficiently high, two spectral windows are sufficient to distinguish more than two, in theory an arbitrarily large number of, different fluorescent probes ${ }^{14}$. The classical implementation of ratiometric single-molecule imaging inserts a dichroic beamsplitter into the fluorescence beam path to create these two spectral detection windows. We realized that the main dichroic beamsplitter used in most fluorescent microscopes to separate the illumination from the fluorescence light already represents two spectral windows: the main transmitted, longer-wavelength component (conventional fluorescence) and a small but non-negligible reflected fraction (Fig. 1a and Supplementary Fig. 1). Salvaging this reflected fluorescence (salvaged fluorescence) provides previously lost spectral information which can be used to assign the molecular identity of an emitter. This approach takes advantage of the fact that spectral assignment and spatial localization precision utilize the fluorescent signal very differently. The former takes advantage of differences between probe spectra, which, given the steep rising edge of the emission spectra, are detected very clearly in the reflected spectral window. This suggests that the salvaged fluorescence window can be quite narrow. The latter depends on the total photon number which, with a narrow salvaged fluorescence window, is mostly collected in the conventional fluorescence channel. Molecules can then be localized based on this channel alone avoiding the need for accurate registration and chromatic corrections necessary in classical ratiometric imaging which combines the signal of both channels. Simulations showed that with a transition edge between windows in the 660-670 nm range, dyes excitable at $642 \mathrm{~nm}$ and suitable for SMS ${ }^{18}$ can be well separated (cross-talk $1 \%$ to $2 \%$, rejection fractions $<1 \%$ to $10 \%)$ with only minor compromises $(\sim 1 \mathrm{~nm})$ in localization precision (Supplementary Fig. 2).

We implemented the SF approach in a 4Pi-SMS instrument (Fig. 1a and Supplementary Fig. 1) and tested it with five far-red dyes (Alexa Fluor 647 (AF647), CF660C, Dyomics 634 (DY634), Dylight 650 (DL650), CF680) for two- and three-color imaging (Fig. 1 and Supplementary Fig. 3). For two-color imaging with AF647 and CF660C (Fig. 1b-f and Supplementary Video 1), we achieved a localization precision ( $\sim \mathrm{nm}$ in xy, $\sim 5 \mathrm{~nm}$ in $\mathrm{z}$ ) that is comparable to the previously reported one-color 4Pi-SMS imaging ${ }^{8}$ and Fourier Shell Correlation (FSC) ${ }^{21}$ values of 25 and $22 \mathrm{~nm}$, respectively (Supplementary Fig. 4a-f).

Consistent with these high localization precision values, the microscope can resolve the hollow center of labeled ER tubules ( $100 \mathrm{~nm}$ in diameter) and microtubules ( $\sim 5 \mathrm{~nm}$ in diameter: $26 \mathrm{~nm}$ actual diameter plus $\sim 10 \mathrm{~nm}$ displacement on either side caused by using antibodies) (Fig. 1k-o). The distinct ratios of salvaged to conventional fluorescence between the two dyes yielded a cross-talk of < 1\% (Fig. 1d,e and Supplementary Fig. 4g,h). The average chromatic shifts between the two channels were determined to be less than $2 \mathrm{~nm}$ in all directions without applying any chromatic corrections (Fig. If and Supplementary Fig. $4 \mathrm{i}-\mathrm{k})$. 
Using DY634, DL650 and CF680 for three-color imaging (Fig. 1g-j), we resolved the tubular structure of immunolabeled microtubules in all three color channels (FSC values DY634: 32 nm, DL650: 25 nm, CF680: 28 nm; Supplementary Fig. 5a-i), and achieved s $2 \%$ cross-talk between each dye pair (Fig. 1i and Supplementary Fig. 5j,k). The average chromatic shifts between the three dyes were determined to be mostly in the 1-3 nm range with the biggest value being $<5 \mathrm{~nm}$ for the dyes with the farthest shifted spectra (Fig. $1 \mathrm{j}$ and Supplementary Fig. 51,m).

\section{Multicolor 4Pi-SMS imaging of ER, mitochondria and synaptonemal complexes}

We further tested our SF approach by imaging spatially-close cellular structures with known geometries (Fig. 2). The luminal and membrane markers of ER were resolved with no apparent chromatic aberrations despite the small diameter (Fig. 2a-e, Supplementary Fig. 6a-c and Supplementary Video 2). The high 3D resolution revealed the substantial differences between two immunolabeling approaches: the apparent ER tubule diameter is about $20 \mathrm{~nm}$ smaller when labeled with a nanobody compared to conventional primary and secondary antibodies (Supplementary Fig. 6d-i), showing that using primary and secondary antibodies increased the displacement between labeled target and dyes by $\sim 10 \mathrm{~nm}$, which is consistent with previous reports 22,23 . Mitochondrial double-stranded DNA (dsDNA) was positioned within and clearly isolated from the outer mitochondrial membrane at various depths (Fig. 2f-i, Supplementary Fig. 7a-c and Supplementary Video 3). In contrast, two outer mitochondrial membrane proteins were in close proximity throughout the volume of the sample (Supplementary Fig. 7d-k), confirming that our method results in alignment errors of less than $4 \mathrm{~nm}$ without applying any chromatic corrections, even in thicker volumes.

To demonstrate the power of the SF approach in thicker cells, we imaged the synaptonemal complex (SC) in intact mouse spermatocytes (Fig. 2j-q, Supplementary Fig. 8 and Supplementary Video 4). Our method revealed the twisted helical structure of two synaptonemal scaffold proteins, SYCP1 and SYCP3, throughout the $10-\mu \mathrm{m}$ thick volume (Fig. 2j-o and Supplementary Fig. 8a-d). Furthermore, imagining N- and C-terminally labeled SYCP1 confirmed that the C-terminus is oriented towards the SYCP3 tracks while the $\mathrm{N}$-terminus extends into the central region ${ }^{24}$ (Fig. $2 \mathrm{j}, \mathrm{m}$ ). Imaging these two proteins alongside Lamin B showed the ends of the SCs connecting to the nuclear lamina (Supplementary Fig. 8e,f), confirming previous results ${ }^{25}$. The ends of fully assembled (Fig. 2 p) as well as partially assembled SCs (Fig. 2q) are closely associated with the lamina.

\section{Multicolor 4Pi-SMS imaging of Golgi apparatus}

The minimal cross-talk and negligible chromatic aberrations make the SF approach a powerful tool for cell biology. To test how well it can reveal the location of proteins in complex 3D morphologies that are otherwise only accessible through EM, we imaged two challenging structures which are of central importance to cell biology: the Golgi apparatus and contact sites between the ER and the plasma membrane (PM).

We imaged the immunolabeled Golgi apparatus in HeLa cells in three colors (Fig. 3a-e, Supplementary Fig. 9 and Supplementary Video 5). The cis, medial, and trans regions 
appeared as distinct structures stacked parallel to each other in cross-sections through the imaged Golgi apparatus (Fig. $3 \mathrm{f}-\mathrm{g}$ ). While the cis-medial-trans stacking was maintained throughout the Golgi, some stacks flipped their orientation within a few hundred nanometers (Fig. 3f, compare dashed line and cyan arrow). The high 3D resolution enabled us to characterize the distance between cis, medial and trans cisternae despite the convoluted morphology of the Golgi apparatus (Fig. 3h-i). The trans-localized p230 and cis-localized GRASP65 labels showed a peak-to-peak distance of on average $187 \mathrm{~nm}$, ranging from 141 $\mathrm{nm}$ (10th percentile) to $236 \mathrm{~nm}$ (90th percentile). This broad distribution of the peak-to-peak distances appeared to consist of two populations which suggests that we observed Golgi stacks with different numbers of cisternae. The medial-localized ManII was on average 85 $\mathrm{nm}$ (10th percentile: $50 \mathrm{~nm}$; 90th percentile: $116 \mathrm{~nm}$ ) apart from GRASP65. In contrast, GRASP65 and the also cis-localized GM130 showed an average separation not significantly different from zero $(P=0.62)$ (Supplementary Fig. 10), confirming previous biochemical data $^{26}$. It has been previously reported that each Golgi cisterna is about $50 \mathrm{~nm}$ thick ${ }^{1}$. Side profile measurements of our data showed that both p230 and GM130 stainings had an average thickness of $77 \mathrm{~nm}$ and $86 \mathrm{~nm}$, respectively (Fig. 3h,j). This is an overestimate as we averaged across $1 \mu \mathrm{m}$ subregions and did not account for the fact that the cisternae are not perfectly flat over these regions. Considering the $\sim 10 \mathrm{~nm}$ label size added by antibody labeling on each side of a cisterna (Supplementary Fig. 6i), this data suggests that p230 and GM130 are enriched in one cisterna only, as expected. Our GRASP65 staining showed a similar thickness as p230 and GM130 (Fig. 3j), indicating that our GRASP65 labeling is concentrated in one cisterna. In contrast, ManII-GFP appeared at an average thickness of $127 \mathrm{~nm}$ implying that it is distributed over multiple cisternae (Fig. 3j).

\section{Multicolor 4Pi-SMS imaging of ER-PM contact sites}

Unlike the perinuclear Golgi, the ER spreads and branches throughout the volume of mammalian cells making contacts with most organelles, including the PM. To gain insight into fine details of ER-PM contacts, where the intermembrane distance is typically within the 15-25 nm range ${ }^{27}$, we imaged the PM (labeled with WGA) and ER membranes in COS-7 cells (Fig. 4 and Supplementary Video 6). At the periphery of the cells, ER tubules are generally clearly separated by tens of nanometers from the top and bottom PM (Fig. 4a). Upon overexpression of an ER protein, ORP5, which functions as a tether at ER-PM contact sites, however, a large fraction of the peripheral ER became closely apposed to the PM to form large, patch-like contacts ${ }^{28}$ (Fig. 4b, cyan arrow). Likewise, overexpression of another ER-PM contact site protein, E-Syt2 (ref. ${ }^{29}$ ), also expanded appositions of ER with the PM, although in this case, the ER retained a tubular shape at such appositions (Supplementary Fig. 11a). This was consistent with E-Syt2 being anchored to the ER membrane by an Nterminal hairpin domain that may sense/induce high-curvature membranes ${ }^{29}$, while ORP5 is anchored to the ER by a single C-terminal transmembrane region ${ }^{28}$. To visualize the contact site proteins directly, we imaged ORP5 or E-Syt2 together with the PM marker (Fig. 4c-f and Supplementary Fig. 11b-f). In agreement with the results shown above, ORP5 appeared as patches (Supplementary Fig. 11b), while E-Syt2 mostly appeared as tubule-like structures (Supplementary Fig. 11c) and occasionally as small patches (Supplementary Fig. 11d). Twocolor imaging further confirmed that the observed ORP5 and E-Syt2 structures corresponded to ER elements (Supplementary Fig. 12a-f). The intensity profile of each cross-section 
showed a small separation between ORP5 or E-Syt2 and the PM (Fig. 4d,f and

Supplementary Fig. 11f, blue arrow). Quantification showed distances of the PM signal peak to the ORP5 and E-Syt2 signal peaks of 15-20 nm (Fig. 4g), respectively, consistent with ESyt1 and E-Syt3 values derived from EM images ${ }^{27}$ and demonstrating the power of SF in resolving ultrastructural details. While it is difficult to visualize specific contact site proteins in EM, our approach allows imaging the ER membrane, contact site proteins, and the PM at the same time. Three-color imaging of ER-PM contact sites showed both contact site proteins as expected at the interface between ER membranes and the PM: ORP5 at patchlike contacts (Fig. 4h,i and Supplementary Fig. 12g) and E-Syt2 at tubular contacts (Fig. 4j,k and Supplementary Fig. 12h). In addition, both ORP5 and E-Syt2 localized to the ER membrane facing the PM but not the other side (Fig. 4h-k and Supplementary Video 7).

\section{Discussion}

The development of high-quality multicolor imaging at tens of nanometers resolution in 3D provides a tool to the cell biologist that combines the strength of specific labeling of fluorescence microscopy in the context of interaction partners and cellular landmarks with a level of detail that traditionally had been the realm of EM.

As we routinely achieve better than $10 \mathrm{~nm}$ localization precision, other factors, such as localization density, drift, chromatic aberrations and localization accuracy become more important ${ }^{3}$. As shown above, chromatic aberrations using our SF approach are below $5 \mathrm{~nm}$, typically in the 1-3 nm range. Using redundant cross-correlation based drift correction, residual drift is reduced to below $5 \mathrm{~nm}^{30,31}$. These low values combined with high localization densities allow us to achieve excellent 22-32 nm FSC values in our microtubule data (Supplementary Figs. 4 and 5).

The localization accuracy (representing the systematic offset of the determined positions from the true positions of target proteins) suffers from the size of the label. In most of the imaging shown in this work, we used antibody labeling (primary + secondary) which creates an average displacement of $\sim 10 \mathrm{~nm}$ from the targets. Using overexpressed GFP labeled with anti-GFP nanobody reduces this displacement to $2-6 \mathrm{~nm}(4 \mathrm{~nm}$ size of GFP $\pm 2 \mathrm{~nm}$ size of nanobody) and results in smaller diameters of the ER tubules (Supplementary Fig. 6i). To further improve the localization accuracy, genetically encoded self-labeling tags (HaloTag ${ }^{32}$ and SNAP-tag ${ }^{33}$ ) or click-chemistry ${ }^{34}$ can be used.

It is important to note that our SF approach with minor optical modifications can also be implemented in single-objective systems (Supplementary Fig. 13a,b), where chromatic focal shifts can be even stronger than in the 4Pi configuration. To show the feasibility, we blocked the top emission beam path in our 4Pi-SMS microscope to mimic the detection of a singleobjective system (Supplementary Fig. 13c). We obtained two-color images with excellent quality in both 2D and 3D with astigmatism (Supplementary Fig. 13d-j). Additionally, the concept can be adopted in many multicolor imaging scenarios, including other singlemolecule imaging techniques and single-particle tracking, or in systems optimized for large field-of-view imaging with uniform illumination ${ }^{35,36}$. 
Similar to classical ratiometric approaches, the ability of our SF approach to image multiple dyes simultaneously, in theory, can reduce the acquisition time. However, in practice, the acquisition speed is often limited by the need to avoid the spatial overlap of blinking molecules which puts a constraint on the maximum number of blinking molecules per frame. Given that multicolor imaging at super-resolution is usually most helpful to reveal the relationship between multiple molecular species in close proximity, molecules from different stainings, which in ratiometric imaging are localized from the same camera images, should ideally not appear at the same time if spatially close to each other. This requires the blinking frequencies of the individual molecular species to be reduced compared to imaging only one color at a time, negating any potential improvement in imaging speed gained by simultaneous multicolor imaging.

We achieve low chromatic shifts of 1-5 nm without any chromatic corrections applied in post-processing and independent of how deep we image in a cell. This stands in contrast to typically 10-20 nm shift between colors when using classic ratiometric approaches, even after applying chromatic corrections in post-processing 16,17 . This advantage is an inherent feature of our SF approach where different dyes are localized from the same camera image using the same spectral detection window. Typical separations between peak emission wavelengths of $20 \mathrm{~nm}$ or more thereby are reduced to only 5-9 $\mathrm{nm}$ in apparent wavelength difference (Supplementary Fig. 3c-g). To eliminate this spectral shift, an alternative approach is sequentially imaging the same dye by washing out one label and replacing it with a second one between imaging sessions ${ }^{37,38}$. These approaches, however, are only compatible with fixed samples.

While only fixed samples are shown in this work, we would like to emphasize the great potential of the SF approach for live-cell imaging (Supplementary Note 2). A preliminary test using two live-cell compatible photoactivatable fluorescent dyes, PA-JF646 and PAJF669, demonstrates that these two dyes can be well separated by the SF approach. Simulations further showed that our approach is compatible with photoactivatable or photoswitchable fluorescent proteins to achieve three-color imaging in live cells with low cross-talk. In concert with other recent developments of live-cell compatible blinking probes $^{34}$, we believe that our SF approach will be a key to successful multicolor live-cell SMS since it eliminates the need for sequential imaging and decreases phototoxicity and photobleaching by using one excitation laser.

\section{Methods}

\section{Synaptonemal complex samples}

All experimental procedures involving the use of mice were performed in agreement with the Yale University Institutional Animal Care and Use Committee (IACUC). BALB/cJ mice (The Jackson Laboratory, Stock No: 000651) were purchased from The Jackson Laboratory. Testes (tunica removed) from 18-day old mice were disrupted using forceps and a razor blade in $1 \mathrm{~mL}$ of PBS (1× PBS; Gibco, Cat\# 10010023) with protease inhibitors (Roche, Complete Ultra, Cat\# 05896988001). The cell suspension was then gently added to a $15-\mathrm{mL}$ conical tube with $5 \mathrm{~mL}$ of $1 \times$ PBS with protease inhibitors and allowed to settle. After approximately 3 minutes, $51-\mathrm{mL}$ aliquots of the cell suspension were placed in $1.5-\mathrm{mL}$ 
microcentrifuge tubes and centrifuged at 9k RPM for 10 minutes. The supernatant was then aspirated, and the pellets were combined in $0.5 \mathrm{~mL}$ of $1 \times$ DPBS (made from 10x stock; Gibco, Cat\#14080-055) per testes. 50-100 $\mu \mathrm{L}$ of the cell suspension was added to \#1.5, 25$\mathrm{mm}$ diameter round precision coverglass, which had been cleaned and Poly-L-Lysine coated (Sigma-Aldrich, Cat\# P4707), and allowed to sit for 30 minutes. The cells on coverglass were then fixed in 4\% paraformaldehyde (PFA; Electron Microscopy Sciences, Cat\# 15710) for 15 minutes at room temperature. Standard immunolabeling was performed using the antibodies listed in the Key Resource Table (See synaptonemal complex labeling section).

\section{Cell culture}

COS-7 and HeLa cells were grown in DMEM or DMEM/F12 (Gibco, Cat\# 21063029 and 21041025 ) supplemented with $10 \%$ fetal bovine serum (Gibco, Cat\# 10438026) at $37{ }^{\circ} \mathrm{C}$ with $5 \% \mathrm{CO}_{2}$. Some cultures were grown with media supplemented with sodium pyruvate (Gibco, Cat\# 11360070).

\section{Plasmids}

For labeling the ER membrane, we expressed the plasmid mEmerald-Sec61-C-18, a gift from Michael Davidson (deceased, formerly Florida State University, Tallahassee, FL; Addgene plasmid \# 54249; which encodes GFP-Sec61 $\beta$ ). For labeling mitochondria, we expressed GFP-OMP25 from a plasmid that was made by modifying pEGFP-C1 (Takara Bio Inc.) to have eGFP fused to the C-terminus of human OMP25/SYNJ2BP cDNA containing the amino acids "QVQNGPIGHRGEGDPSGIPIFMVLVPVFALTMVAAWAFMRYRQQL" and localizes to mitochondria, as shown previously ${ }^{39}$. For labeling the medial Golgi, we expressed GFP-ManII from a plasmid that was made from pEGFP-N1 (Takara Bio Inc.) to have amino acids 1-137 of mouse Man2a1 fused to GFP, such that GFP is located in the Golgi lumen. For labeling the ER lumen, we expressed mCherry-KDEL from a plasmid that was made by modifying pDsRed2-ER (Takara Bio Inc.), which encodes a signal peptide fused to DsRed2 followed by the tetrapeptide ER retention signal KDEL. The mCherry gene was amplified using the following primers 5'-

ATACCGGTCGATGGTGAGCAAGGGCGAG-3' and 5'CTGAAGCTTTTACAGCTCGTCCTTCTTGTACAGCTCGTCCATGCC-3'. The pDsRed2ER plasmid and the mCherry PCR product were both digested using AgeI and HindIII (New England Biolabs, Cat\# R0552S and R3104S) and ligated together, replacing the DsRed2 gene with the mCherry gene. Plasmids encoding GFP-ORP5 and mCherry-ORP5 were previously published ${ }^{28}$. Plasmids encoding mCherry-E-Syt2 and GFP-E-Syt-2 were previously published ${ }^{29}$.

\section{Coverglass cleaning}

Precision thickness coverglass (Bioscience tools, Cat\# CSHP-No1.5-25) was cleaned before cells were plated on them. Before plating COS-7 cells, glass was cleaned in a sonic bath (Bronson) immersed in $1 \mathrm{M} \mathrm{KOH}$ for 15 minutes and then rinsed with MilliQ water three times. Glass was then sterilized with 100\% Ethanol, incubated with Poly-L-Lysine for 10 minutes, then rinsed with sterile PBS before adding media and cells. Before plating HeLa cells, glass was instead cleaned with an ozone cleaner (Jelight, UVO Cleaner Cat\# 342A) for 30 minutes. Media and HeLa cells were placed directly on ozone-cleaned glass. Before 
placing spermatocytes, glass was cleaned in a plasma oven for 5 minutes before being coated with Poly-L-Lysine.

\section{Transfection}

Samples including GFP-Sec61 $\beta$, mCherry-KDEL and GFP-OMP25 expression in HeLa or COS-7 cells used DNA transfection by electroporation. DNA was introduced to HeLa or COS-7 cells using a NEPA GENE electroporation device. Approximately 1 million cells were rinsed in Opti-MEM (Gibco, Cat\# 31985070) and then resuspend in Opti-MEM with $10 \mu \mathrm{g}$ DNA in an electroporation cuvette with 2-mm gap (Bulldog Bio, Cat\# 12358346). Cells were electroporated with a poring pulse of $125 \mathrm{~V}, 3$-ms pulse length, 50-ms pulse interval, 2 pulses, with decay rate of $10 \%$ and + polarity; followed by a transfer pulse of 25 $\mathrm{V}, 50$-ms pulse length, 50-ms pulse interval, 5 pulses, with a decay rate of $40 \%$ and \pm polarity. After electroporation, the cells were removed from the cuvette and grown in growth media on prepared coverglass. Samples were fixed 18-24 hours after electroporation (see Methods, coverglass cleaning section).

Samples including ER-PM contact site protein expression in COS-7 cells utilized Lipofectamine2000 (Invitrogen, Cat\# 11668027) transfection. The day before transfection, cells were plated on prepared coverglass. Transfection was performed as suggested by the manufacturer with a modification of $1 \mu \mathrm{L}$ transfection reagent and $1 \mu \mathrm{g}$ DNA per well in a 6well plate. When two DNAs were transformed together, $0.5 \mu \mathrm{g}$ of each DNA was used for a total of $1 \mu \mathrm{g}$ DNA. We did not find that different transfection approaches affect the image quality.

\section{Fluorescent dyes}

Alexa Fluor 647 (AF647) and CF660C were used for all two-color imaging experiments except for the ER-PM imaging where CF680 was used instead of CF660C (see Methods, ER-PM contact sites labeling). Secondary antibodies labeled with AF647 (Invitrogen, Cat\# A21236, A21237, or A21245; used at 1:1000 dilution for 1 hour at room temperature) and

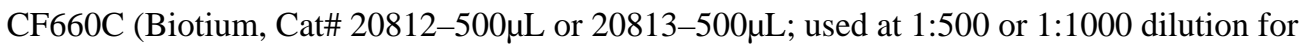
1 hour at room temperature) were used to label primary antibodies. These CF660C-labeled antibodies were manufactured to have one dye per antibody. Nanobody GFP-binding protein (Chromotek, Cat\# gt-250) and RFP-binding protein (Chromotek, Cat\# rt-250) were conjugated to AF647 in lab (see Methods, nanobody and antibody conjugation section).

Dyomics634 (DY634), DyLight 650 (DL650), and CF680 were used for all three-color imaging experiments. Secondary antibodies labeled with DY634 (conjugated in lab, see nanobody and antibody conjugation section; used at 1:200 dilution for 1 hour at room temperature), DL650 (Invitrogen, Cat\# SA5-10174 or SA5-10034; used at 1:1000 dilution for 1 hour at room temperature), and CF680 (Biotium, Cat\# 20817-500 $\mu \mathrm{L}$ or 20818-500 $\mu \mathrm{L}$; used at 1:500 or 1:1000 dilution for 1 hour at room temperature) were used to label primary antibodies. The CF680-labeled antibodies were manufactured to have one dye per antibody. Nanobody RFP-binding protein conjugated to DY634 and nanobody GFP-binding protein conjugated to DL650 were made in lab (see Methods, nanobody and antibody conjugation section). WGA-CF680 (Biotium, Cat\# 29029-1) was used for plasma membrane labeling. 


\section{Nanobody and antibody conjugation}

Nanobody RFP-binding protein or GFP-binding protein was conjugated to Alexa Fluor 647 NHS-ester (Life Technologies, Cat\# A20006), DY634 NHS-ester (Dyomics, Cat\# DY-634NHS-ester portionized, 634-01A), or DL650 NHS-ester (Thermo Scientific, Cat\# 62265). Approximately $100 \mu \mathrm{L}$ conjugations were performed in 0.1 M Sodium Bicarbonate for 1 hour in the dark. Excess dye was removed from the conjugation reaction using Zeba Spin Desalting Columns with a 7K molecular weight cut off (Thermo Scientific, Cat\# 89882). Nanobodies were used at 1:1000 dilution at room temperature or overnight at $4{ }^{\circ} \mathrm{C}$.

Unlabeled goat anti-rabbit IgG, and goat anti-mouse IgG, and goat anti-human IgG (Jackson ImmunoResearch, Cat\# 111-005-144, 115-005-146, and 109-005-088, respectively) were conjugated with DY634 NHS ester (Dyomics, DY-634-NHS-ester portionized, 634-01A). Approximately $100 \mu \mathrm{L}$ reactions were performed in $0.1 \mathrm{M}$ Sodium Bicarbonate for 1 hour in the dark. Excess dye was removed from antibody using Pro-Spin columns (Princeton Separations, Cat\# CS800) per manufacturer's recommendations. Secondary antibodies conjugated in this work were used at 1:200 for 1 hour at room temperature.

\section{Sample labeling}

Microtubule samples-To label microtubules, COS-7 cells were prepared as previously reported ${ }^{8}$. After growing on Poly-L-Lysine coated coverglass for 24 hours, cells were rinsed with PBS, warmed to $37^{\circ} \mathrm{C}$, then incubated for 1 minute in $0.05 \%$ saponin diluted in cytoskeletal buffer (CBS; $10 \mathrm{mM}$ MES pH 6.1, $138 \mathrm{mM} \mathrm{NaCl}, 3 \mathrm{mM} \mathrm{MgCl} 2,2 \mathrm{mM}$ EGTA, $320 \mathrm{mM}$ sucrose), warmed to $37^{\circ} \mathrm{C}$. Cells were subsequently fixed in $3 \%$ paraformaldehyde and $0.1 \%$ glutaraldehyde (GA; Electron Microscopy Sciences, Cat\# 16019) diluted in CBS, warmed to $37{ }^{\circ} \mathrm{C}$. Cells were permeabilized and blocked with $3 \%$ bovine serum albumin (BSA; Jackson ImmunoResearch, Cat\# 001-000-162) and 0.2\% Triton X-100 (TX-100; Sigma-Aldrich, Cat\# T8787) in 1× PBS (diluted from 10× PBS; American Bio, Cat\# $\mathrm{AB} 11072-0100)$ for 30 minutes. The samples were incubated overnight at $4{ }^{\circ} \mathrm{C}$ with mouse anti-a-tubulin antibody (Sigma-Aldrich, Cat\# T5168) diluted to 1:200 in antibody dilution buffer (1\% BSA and $0.2 \%$ TX-100 in $1 \times$ PBS). Cells were washed in wash buffer $(0.05 \%$ TX-100 in $1 \times$ PBS) three times for 5 minutes each. For single-color labeling, microtubule samples were incubated with each dye-conjugated secondary antibody (used at 1:1000) in antibody dilution buffer for 1 hour at room temperature then washed three times for 5 minutes with wash buffer. For two- or three-color labeling, microtubule samples were incubated with the dye-conjugated secondary antibodies (used at 1:1000) together. Lastly, the samples were post-fixed in 3\% PFA $+0.1 \%$ GA in CBS for 10 min, rinsed with PBS three times, and stored in PBS at $4{ }^{\circ} \mathrm{C}$ until imaged.

ER and microtubule labeling-COS-7 cells overexpressing GFP-Sec61 $\beta$ and were grown on Poly-L-Lysine coated glass for 24 hours before being fixed with 3\% PFA $+0.1 \%$ GA in PBS for 15 minutes at room temperature. Samples were rinsed three times in $1 \times$ PBS before permeabilizing for 3 minutes using permeabilization buffer (PB; 0.3\% CA-630 (Sigma-Aldrich, Cat\# I8896), 0.05\% TX-100, 0.1\% BSA, and 1× PBS) at room temperature. Samples were then rinsed three times with $1 \times$ PBS followed by 1 hour in blocking buffer (BB; 0.05\% CA-630, 0.05\% TX-100, 5\% normal goat serum (Jackson ImmunoResearch, 
Cat\# 005-000-121), and 1× PBS). Primary antibodies, rabbit anti-GFP (Invitrogen, Cat\# A11122, used at 1:500) and mouse anti-tubulin (used at 1:1000), were diluted in BB and incubated with samples overnight at $4{ }^{\circ} \mathrm{C}$. Samples were then washed in wash buffer (WB; $0.05 \%$ CA-630, $0.05 \% \mathrm{TX}-100,0.2 \% \mathrm{BSA}$, and $1 \times \mathrm{PBS}$ ) three times for 5 minutes each before secondary antibody labeling for 1 hour at room temperature diluted in BB. Samples were washed in WB three times for 5 minutes each. Post-fixation was performed using 3\% PFA $+0.1 \%$ GA in $1 \times$ PBS for 10 minutes. Samples were rinsed three times in $1 \times$ PBS before being stored in $1 \times \mathrm{PBS}$ at $4{ }^{\circ} \mathrm{C}$.

ER membrane and ER lumen labeling-COS-7 cells overexpressing GFP-Sec61 $\beta$ alone or GFP-Sec61 $\beta$ and mCherry-KDEL together were plated on cleaned and Poly-LLysine coated glass and grown for 24 hours before being fixed in $3 \%$ PFA $+0.1 \%$ GA in $1 \times$ PBS for 15 minutes.

All ER samples were permeabilized with PB (0.3\% CA-630, 0.05\% TX-100, 0.1\% BSA, and $1 \times \mathrm{PBS}$ ) for 3 minutes at room temperature, rinse in $1 \times$ PBS three times, and then blocked in BB (0.05\% CA-630, 0.05\% TX-100, 5\% normal goat serum, and $1 \times$ PBS) for an hour. Samples were washed in WB (0.05\% CA-630, 0.05\% TX-100, 0.2\% BSA, and 1× PBS) three times for 5 minutes each after labeling with primary antibody, secondary antibody, or nanobody. The ER was labeled using different combinations of antibodies and/or nanobodies.

For two-color ER membrane labeling with antibodies, GFP-Sec61 $\beta$ was labeled with rabbit anti-GFP which was then labeled with two competing secondary antibodies. For two-color ER membrane labeling combining nanobody and antibody, GFP-binding protein nanobody, conjugated with AF647, first was used to label GFP-Sec61 $\beta$ at room temperature for 1 or 2 hours. Then rabbit anti-GFP (used at 1:500) was incubated with samples at $4{ }^{\circ} \mathrm{C}$ overnight, which was subsequently labeled with CF660C. Two-color ER membrane and lumen labeling was performed on cells expressing GFP-Sec61 $\beta$ and mCherry-KDEL. Lumen-localized mCherry-KDEL was first labeled using RFP-binding protein nanobody conjugated to AF647 for 2 hours at room temperature. GFP-Sec61 $\beta$ was then labeled using rabbit anti-GFP (used at 1:500 at $4{ }^{\circ} \mathrm{C}$ overnight) which was then labeled with CF660C. All samples were postfixed using 3\% PFA $+0.1 \%$ GA in $1 \times$ PBS for 10 minutes. Samples were rinsed three times in $1 \times$ PBS before being stored in $1 \times$ PBS at $4{ }^{\circ} \mathrm{C}$.

Mitochondria labeling - HeLa cells were used for imaging mitochondria. For some samples, GFP-OMP25 was overexpressed for 18-24 hours. Mitochondria samples were fixed differently depending on the primary antibody being used, with a preference for $3 \%$ PFA $+0.1 \%$ GA in $1 \times$ PBS for 15 minutes at room temperature. Samples that included mouse anti-dsDNA antibody labeling, since this primary antibody does not label cells if GA is used during fixation, were fixed using $4 \%$ PFA in $1 \times$ PBS for 1 hour at room temperature. All cells were permeabilized with PB $(0.3 \%$ CA-630, $0.05 \%$ Triton X-100, 0.1\% BSA, and $1 \times$ PBS) for 3 minutes at room temperature, rinsed three times in PBS, and then blocked in BB $(0.05 \%$ CA-630, $0.05 \%$ TX-100, 5\% normal Goat serum, and $1 \times$ PBS). 
Primary antibodies were diluted in BB and incubated with samples according to the antibody used. For outer-mitochondria/outer-mitochondria 2-color samples, mouse anti-GFP (Invitrogen, Cat\# A11120, used at 1:500) was incubated on samples at $4{ }^{\circ} \mathrm{C}$ overnight followed by rabbit anti-TOM20 (Abcam, Cat\# ab78547, used at 1:1000) on samples at room temperature for 1 hour. For outer-mitochondria/nucleoid two-color samples, mouse antidsDNA (Abcam, Cat\# ab27156, used at 1:1000) was incubated with samples at $4{ }^{\circ} \mathrm{C}$ overnight followed by rabbit anti-TOM20 (used at 1:500) on samples at room temperature for 1 hour the following day. When labeling inner and outer mitochondria, the inner mitochondria primary and secondary labeling was completed before beginning labeling the outer mitochondria. After each antibody labeling, samples were washed in WB $(0.05 \%$ CA-630, 0.05\% TX-100, 0.2\% BSA, and $1 \times$ PBS) three times for 5 minutes. Two-color samples were labeled with AF647 and CF660C secondary antibodies. Post-fixation was performed using 3\% PFA $+0.1 \%$ GA in PBS for 10 minutes. Samples were rinsed three times in PBS before being stored in PBS at $4{ }^{\circ} \mathrm{C}$.

Synaptonemal complex labeling-After being fixed with 4\% PFA, the samples were then washed with $1 \times$ PBS 3 times followed by a permeabilization step using $0.5 \%$ TX-100 in $1 \times$ PBS for 10 minutes at room temperature. Samples were then rinsed in 0.1\% TX-100 in PBS and treated with Image-iT Signal Enhancer (Molecular Probes, Cat\# I36933) for 30 minutes at room temperature. After 3 washes in $0.1 \%$ TX-100 in PBS, the samples were incubated in blocking buffer $(0.05 \% \mathrm{TX}-100,5 \%$ normal goat serum, $0.05 \%$, in $1 \times \mathrm{PBS})$ for 30 minutes at room temperature. For SYCP3 and SYCP1-C (or SYCP1-N) two-color samples, rabbit anti-SYCP1-C (Novus Biologicals, Cat\# N300-229, used at 1:500) (or rabbit anti-SYCP1-N ${ }^{24}$, a gift from Dr. Ricardo Benavente of University of Wuerzburg, used at 1:500) was incubated with samples at $4{ }^{\circ} \mathrm{C}$ overnight followed by mouse anti-SYCP3 (Abcam, Cat\# ab97672, used at 1:500) on samples at room temperature for 1 hour the following day. After each primary antibody labeling, samples were washed in WB $(0.05 \%$ CA-630, 0.05\% TX-100, 0.2\% BSA, and $1 \times$ PBS) three times for 5 minutes. Two-color samples were labeled with AF647 and CF660C secondary antibodies. For SYCP3/SYCP1$\mathrm{C} /$ Lamin B three-color samples, rabbit anti-SYCP1-C (used at 1:500) was incubated with samples at $4{ }^{\circ} \mathrm{C}$ overnight. The next day mouse anti-SYCP3 (used at 1:500) was incubated with samples at room temperature for 1 hour followed by a 1-hour incubation at room temperature with chicken anti-Lamin B (Abcam, Cat\# ab90169, used at 1:200). After each primary antibody labeling, samples were washed in WB (0.05\% CA-630, 0.05\% TX-100, $0.2 \%$ BSA, and $1 \times$ PBS) three times for 5 minutes. Three-color samples were labeled with CF680 (used at 1:1000), DY650 (used at 1:1000), and Dyomics 634 (used at 1:200) secondary antibodies. For synaptonemal complex samples, we performed the imaging when they were freshly made and avoided the extra washing steps associated with post-fixation which may remove cells from the sample. Upon entry into meiosis, the nuclear lamina is disrupted leading to the fragmented appearance shown in Fig. 2 and Supplementary Video 4.

Golgi labeling-Since we did not identify a good anti-ManII antibody suitable for immunolabeling, we electroporated HeLa cells with a plasmid encoding ManII-GFP to label the medial Golgi apparatus. Cells were transferred to cleaned coverglass and expressed the plasmid for 18-24 hours before being fixed with 4\% PFA in $1 \times$ PBS for 15 minutes at room 
temperature. Cells were permeabilized with PB (0.3\% CA-630, 0.05\% TX-100, 0.1\% BSA, and $1 \times$ PBS) for 3 minutes at room temperature, rinsed three times in PBS, and then blocked in $\mathrm{BB}(0.05 \%$ CA- $630,0.05 \%$ TX-100, $5 \%$ normal Goat serum, and $1 \times$ PBS).

Primary antibodies were diluted in BB and incubated with samples according to the antibodies used. For cis/medial/trans labeled samples, mouse anti-p230 (BD Bioscience, Cat\# 611280, used at 1:1000) and nanobody GFP-binding protein, conjugated with DL650, were incubated with samples at $4{ }^{\circ} \mathrm{C}$ overnight followed by rabbit anti-GRASP65 (Abcam, Cat\# ab174834, used at 1:2000) on samples for 1 hour at room temperature the next day. Alternatively, for cis/cis/medial labeled samples, mouse anti-GM130 (BD Bioscience, Cat\# 610822, used at 1:500) and nanobody GFP-binding protein, conjugated with DL650, were incubated on samples together at $4{ }^{\circ} \mathrm{C}$ overnight followed by rabbit anti-GRASP65 (used at 1:3000) on samples for 1 hour at room temperature the next day. After each antibody labeling, samples were washed in WB $(0.05 \%$ CA-630, $0.05 \%$ TX-100, $0.2 \%$ BSA, and $1 \times$ PBS) three times for 5 minutes. After labeling with both primary antibodies and nanobody, samples were incubated with secondary antibodies labeled with DY634 and CF680 together. Samples were post-fixed using 3\% PFA $+0.1 \%$ GA in PBS for 10 minutes. Samples were rinsed three times in PBS before being stored in PBS at $4{ }^{\circ} \mathrm{C}$.

ER-PM contact sites labeling-COS-7 cells were grown on Poly-L-Lysine coated glass and transfected with plasmids encoding the following proteins: GFP-Sec61 $\beta$, GFP-ORP5, mCherry-ORP5, GFP-E-Syt2, or mCherry-E-Syt2 (in different combinations) using Lipofectamine2000. Cells were fixed with $3 \%$ PFA $+0.1 \%$ GA $18-24$ hours after transfection. If the plasma membrane was lectin-labeled, the samples were labeled directly after fixation but before permeabilization. Cells were rinsed with Hanks balanced salt solution (HBSS; Gibco, Cat\# 14025-092) three times before labeling with $1 \mu \mathrm{g} / \mathrm{mL}$ WGACF680 (Biotium, 29029-1) diluted in HBSS for 10-30 minutes at room temperature. Cells were then rinsed three times in HBSS and once in PBS before being permeabilized using PB (0.3\% CA-630, $0.05 \% \mathrm{TX}-100,0.1 \% \mathrm{BSA}$, and $1 \times \mathrm{PBS})$ for 3 minutes at room temperature. Samples were rinsed three times in PBS and blocked for at least 1 hour with BB $(0.05 \%$ CA-630, $0.05 \%$ TX-100, 5\% normal Goat serum, and $1 \times$ PBS). Primary antibodies and/or nanobodies in BB were incubated with samples overnight at $4{ }^{\circ} \mathrm{C}$.

For two-color samples labeling ER and PM (expressing GFP-Sec61 $\beta$ ) and two-color samples labeling contact proteins and PM (expressing GFP-ORP5 or GFP-E-Syt2), GFP was labeled with rabbit anti-GFP (used at 1:500 overnight at $4{ }^{\circ} \mathrm{C}$ ) followed by a secondary AF647 antibody. Some ER and PM samples also expressed mCherry-ORP5 or mCherry-ESyt2, which were not immunolabeled. For two-color samples labeling ER and contact proteins (expressing GFP-Sec61 $\beta$ with either mCherry-ORP5 or mCherry-E-Syt2), rabbit anti-mCherry (Abcam, Cat\# ab167453 used at 1:500 or 1:1000 overnight at $4{ }^{\circ} \mathrm{C}$ ) and mouse anti-GFP (used at 1:500 overnight $4{ }^{\circ} \mathrm{C}$ ) were used to label the fluorescent proteins, followed by secondary antibodies labeled with AF647 and CF660C. For three-color samples labeling ER, contact site proteins, and PM (expressing GFP-Sec61 $\beta$ with either mCherryORP5 or mCherry-E-Syt2), WGA was used to label the PM, rabbit anti-mCherry (used at 1:500 or $1: 1000$ overnight at $4{ }^{\circ} \mathrm{C}$ ) and mouse anti-GFP (used at $1: 500$ overnight $4{ }^{\circ} \mathrm{C}$ ) were used to label the fluorescent proteins, followed by secondary antibodies labeled with DY634 
and DL650. Post-fixation was performed using 3\% PFA + 0.1\% GA in PBS for 10 minutes. Samples were rinsed three times in PBS before being stored in PBS at $4{ }^{\circ} \mathrm{C}$.

Mitochondria and microtubule-COS-7 cells were used for two-color labeling of mitochondria and microtubules. Cells were grown on cleaned and Poly-L-Lysine coated glass before being fixed in 3\% PFA $+0.1 \%$ GA in $1 \times$ PBS for 15 minutes. Samples were rinsed three times in PBS before permeabilizing for 3 minutes using permeabilization buffer (PB; 0.3\% CA-630, $0.05 \%$ Triton X-100, 0.1\% BSA, and $1 \times$ PBS) at room temperature. Samples were then rinsed three more times with PBS followed by 1 hour in block buffer (BB; 0.05\% CA-630,0.05\% Triton X-100, 5\% normal Goat serum, and 1× PBS). Primary antibodies rabbit anti-TOM20 (used at 1:500) and mouse anti-tubulin (used at 1:1000) were diluted in BB and incubated with samples overnight at $4{ }^{\circ} \mathrm{C}$. Samples were then washed in wash buffer (WB; $0.05 \%$ CA-630,0.05\% TX-100, 0.2\% BSA, and $1 \times$ PBS,) three times for 5 minutes each before secondary antibody labeling for 1 hour at room temperature diluted in BB. Samples were then washed in WB three times for 5 minutes each. Post-fixation was performed using 3\% PFA $+0.1 \%$ GA in PBS for 10 minutes. Samples were rinsed three times in PBS before being stored in PBS at $4{ }^{\circ} \mathrm{C}$.

Imaging buffer and sample mounting-The conventional $\beta$-mercaptoethanol ( $\beta$ ME) STORM imaging buffer was prepared as previously reported ${ }^{40}$. The imaging buffer was made every time immediately before use where catalase and glucose oxidase were diluted in base buffer (50 mM Tris pH 8.0, $50 \mathrm{mM} \mathrm{NaCl}, 10 \%$ glucose) with the addition of $\beta \mathrm{ME}$ (Sigma-Aldrich, Cat\# M3148-25ML). The final concentration of $\beta M E$ is $143 \mathrm{mM}$. The samples were mounted in a custom-designed sample holder as previously described ${ }^{8}$. Briefly, the sample coverslip was mounted in the sample holder facing up. Then $100 \mu \mathrm{L}$ of imaging buffer was evenly spread on the sample coverslip and a clean coverslip was put on top (attention was given to avoid bubbles trapped between the two coverslips). Excess imaging buffer was drained using Kimwipes. The samples were then sealed with twocomponent silicone glue (Picodent Twinsil, Picodent, Wipperfürth, Germany). After the silicone glue hardened (typically 20-30 min), the samples were transferred to the 4Pi-SMS microscope for imaging. The imaging buffer would usually allow for imaging of about $8-10$ hours.

\section{Multicolor 4Pi-SMS setup}

The multicolor 4Pi-SMS system was built based on the previously described instrument ${ }^{8}$ with minor modifications (Supplementary Fig. 1). The oil immersion objectives were replaced with high numerical aperture (NA) silicone immersion objectives $(100 \times / 1.35 \mathrm{NA}$, Olympus) for better refractive index matching. The system was equipped with two excitation lasers at $560 \mathrm{~nm}$ (MPB Communications, 2RU-VFL-P-2000-560-B1R), $642 \mathrm{~nm}$ (MPB Communications, 2RU-VFL-2000-642-B1R) and an activation laser at $405 \mathrm{~nm}$ (Coherent OBIS $405 \mathrm{LX}, 50 \mathrm{~mW}$ ). Details about the dichroic beamsplitter and emission filters used in the system are shown in Supplementary Fig. 1b. The conventional fluorescence follows the same emission path as the previous design and is collected by a sCMOS camera (ORCAFlash 4.0v2, Hamamatsu) (Supplementary Fig. 1a, Camera 1). The salvaged fluorescence is reflected by the dichroic beamsplitter to the back side of the system and collected by an 
EMCCD camera $(128 \times 128$ pixels, iXon DU860, Andor) (Supplementary Fig. 1c, Camera 2). Both cameras were controlled by custom-written LabVIEW (National Instruments) programs.

\section{Image acquisition}

During image acquisition, the sCMOS camera was set to external trigger mode and the EMCCD camera was set to internal trigger and frame transfer mode. For synchronization, the Fire output of the EMCCD camera was used to trigger the sCMOS camera. The electron multiplication gain of the EMCCD camera was set to 200 for all experiments. Biological samples were imaged at $100 \mathrm{~Hz}$ with a laser $\left(642 \mathrm{~nm}\right.$ ) intensity of about $7.5 \mathrm{~kW} / \mathrm{cm}^{2}$ (twocolor imaging) or $200 \mathrm{~Hz}$ at about $15 \mathrm{~kW} / \mathrm{cm}^{2}$ (three-color imaging). The 405-nm activation laser was manually adjusted to maintain a low density of single molecules per frame. For samples thicker than $1 \mu \mathrm{m}$, the stage was translated at 500-nm steps every 3000 frames for multiple times to cover the entire volume. Typically, 180,000 to 360,000 frames were recorded which corresponds to acquisition times of $15 \mathrm{~min}$ to $1 \mathrm{hr}$.

\section{Image analysis and color assignment}

The images recorded with the sCMOS camera (conventional fluorescence) were analyzed as previously described ${ }^{8}$. Briefly, the lateral positions (xy) of single molecules were determined by fitting with sCMOS-specific algorithms ${ }^{40}$. The phase values (z positions) were estimated from the $0^{\text {th }}$ moment Gaussian intensities of the four images and unwrapped using the metric developed previously ${ }^{8}$. To translate the phase values to axial positions, the intensity modulation frequency for each dye was determined from the simulated 4Pi PSFs using a pupil function-based approach ${ }^{41}$. The simulation was performed using the average wavelength of the detected emission spectra in the conventional fluorescence channel of each dye (Supplementary Fig. 3c-g). For the images recorded with the EMCCD camera (salvaged fluorescence), the readout counts were converted to photoelectrons using the conversion factor provided by the manufacturer. Then the pixel-wise median operation (time window $=3000$ frames) in MATLAB (MathWorks) was used as a filter algorithm to remove the background signals. The positions of localized molecules in the sCMOS images were then mapped to the EMCCD images using a second-order affine transformation matrix obtained by imaging a fluorescent bead sample. The corresponding regions in the EMCCD images were cropped out $(5 \times 5$ pixels, $170 \mathrm{~nm}$ pixel size $)$ and multiplied by a normalized 2D Gaussian distribution with a standard deviation of $130 \mathrm{~nm}$ centered at the positions of the molecules. This Gaussian weighting was performed to reduce the influence from the background regions. The intensity of the molecules in the EMCCD images was calculated by summing the values of the Gaussian-weighted regions. For color assignment, the intensities of the molecules in the salvaged and conventional fluorescence images were plotted on a logarithmic scale (Fig. 1d,h) and binned to a 2D histogram intensity image (Supplementary Fig. 4g and Supplementary Fig. 5j). An appropriate threshold (typically $2 \%$ of each peak value) was applied to separate the different dye molecules with a low cross-talk (Supplementary Fig. 4g,h and Supplementary Fig. 5j,k). After color assignment, the phaseunwrapped values of each dye molecules from the sCMOS images were translated to axial positions using the wavelength-dependent modulation frequencies obtained above.

Localizations $(\mathrm{x}, \mathrm{y}$, and $\mathrm{z}$ ) from all color channels were combined for drift correction using a 
custom algorithm based on redundant cross-correlation $30,31,42$. We performed two iterations: the first round with linear interpolation in each time window followed by a second round without interpolation. The residual drift in each direction is typically less than $5 \mathrm{~nm}$ after correction. For samples requiring axial stepping, multiple optical sections were aligned using a 3D cross-correlation method ${ }^{30}$. Localizations that appear at consecutive frames within a radius of 2 times the localization precision were considered to represent the same molecule and combined. Localized molecules were rejected based on the following criteria: photon number $<500$, lateral localization precision $>25 \mathrm{~nm}$, interference contrast $<0.4$ or log-likelihood ratio $>300$. Particularly, we found the log-likelihood ratio ${ }^{43}$ to be a very effective metric to reduce multi-emitter artifacts. All 4Pi-SMS images and videos were rendered with Vutara SRX software (Bruker). Briefly, the intensity-based images were rendered using Point Splatting mode (10-30 nm particle size), which represents each molecule as a 3D Gaussian distribution with a full-width at half-maximum (FWHM) of the same size. For the overview images, the pixel size is $4-8 \mathrm{~nm}$. For the zoomed-in regions, the pixel size is $2-4 \mathrm{~nm}$.

\section{Thickness and separation measurements of the Golgi cisternae}

For each 3D Golgi volume, we cropped 1- $\mu$ m wide subregions along the $\mathrm{x}$ - or $\mathrm{y}$-direction. We manually reoriented the subregions to generate $2 \mathrm{D}$ projection images providing a side view of the region of the Golgi stack to be analyzed (Fig. 3f,g and Supplementary Fig. 10e,f). Next, we took line profiles (thickness $=100 \mathrm{~nm}$ ) at positions where the three markers are parallel to each other (Fig. $3 \mathrm{f}$ and Supplementary Fig. 10f). Each intensity profile was fit by a Gaussian function to determine the thickness (estimated as the FWHM of the Gaussian distribution when applicable) and the separation between cisternae (distance between peak positions).

\section{Quantification of the ER-PM contact sites images}

The two-color images of the contact site proteins (ORP5 or E-Syt2) and PM (Supplementary Fig. 11b,c) were automatically divided into $100 \times 100 \mathrm{~nm}$ x-y subregions. To ensure a high signal-to-noise ratio, only subregions with more than 100 localizations from the PM labeling (WGA) and 300 localizations from ORP5 (or E-Syt2) were kept for further data analysis (more than 2800 subregions for each condition). The WGA labeling featured much brighter staining of the top PM compared to the bottom PM which resulted in much higher WGA localization densities at the top PM than the bottom PM. We therefore used the top PM signal only for distance measurements. WGA localizations with z positions below the median z positions of ORP5 (or E-Syt2) were rejected as they were considered to belong to the bottom PM. The remaining z positions of WGA and the z positions of ORP5 (or E-Syt2) were binned into histograms (with $5 \mathrm{~nm}$ bin size), respectively. The histograms of WGA and ORP5 (or E-Syt2) localizations were both fit by Gaussian functions to determine the two peak positions and the distance between them (Fig. 4g). In Fig. 4d,f,i,k, the line plots were generated from the corresponding rendered images. Localizations were binned at a pixel size of 3-4 nm (slightly varying depending on the field-of-view) and Gaussian-blurred with a standard deviation of $4.2 \mathrm{~nm}(\mathrm{FWHM}=10 \mathrm{~nm})$. 


\section{Simulation of the SF approach performance}

The salvaged fluorescence signal is mainly determined by the transition wavelength of the dichroic beamsplitter while the conventional fluorescence signal is determined by the spectral window confined by the dichroic beamsplitter and the emission filter (in front of Camera 1, Supplementary Fig. 1a). The performance of the SF approach is therefore expected to depend on the choice of the transition wavelength of the dichroic beamsplitter. To investigate this phenomenon, we performed simulations for transition wavelengths ranging from $650 \mathrm{~nm}$ to $680 \mathrm{~nm}$ (Supplementary Fig. 2). To use realistic simulation parameters, we based our simulation on experimental data. For each dye, we randomly selected 2 million single molecules from real imaging experiments. The photon number of each molecule (determined from the conventional fluorescence channel) was converted to the total photon number representing the whole emission spectrum taking the experimentally used spectral detection window into account. Next, we took the transition profile of a commercially available dichroic beamsplitter (Semrock Di01-R405/488/561/635) which features a steep rising edge in the $\sim 657 \mathrm{~nm}$ range. The transition wavelength of the dichroic beamsplitter was defined as the wavelength at which the transmission is $50 \%$. The transmission profile starts to rise at $650 \mathrm{~nm}$. Below that value, we assumed $2 \%$ transmission. Above $664 \mathrm{~nm}$, we assumed 98\% transmission. For our simulation, we shifted this transition profile across the spectrum starting at a transition wavelength of $650 \mathrm{~nm}$ and ending at 680 $\mathrm{nm}$, with the assumption that the conventional fluorescence channel collects the transmitted signal up to $750 \mathrm{~nm}$ and the salvaged fluorescence channel collects the reflected signal upwards from $645 \mathrm{~nm}$ (based on the used excitation laser wavelength of $642 \mathrm{~nm}$ ). The fraction of conventional and salvaged fluorescence was calculated for each simulation and photon numbers were allocated to each channel accordingly. We then generated synthetic single-molecule images based on these photon numbers in each channel and added background and readout noise similar to experimentally observed values. Finally, these images were analyzed in the same way as experimental data and the cross-talk and localization precisions were calculated taking advantage of the ground truth provided by the simulation and averaged for 2 million simulated molecules each.

We found that less than 10\% of Alexa 647 (when compared to CF660C) and DL650 (when compared to CF680) molecules were rejected at transition wavelengths of $661 \mathrm{~nm}$ or above, and $662 \mathrm{~nm}$ or above, respectively. As expected, localization precision degraded with increasing transition wavelengths since larger transition wavelengths lead to narrower conventional fluorescence spectral windows. Up to $670 \mathrm{~nm}$ transition wavelength, the loss in localization precision stayed below $2 \mathrm{~nm}$, which we considered acceptable. The dichroic beamsplitter used in our microscope (Chroma ZT405/488/561/647rpc) features a transition wavelength of $668 \mathrm{~nm}$ which falls within this acceptable window between $661 \mathrm{~nm}$ or 662 $\mathrm{nm}$ on the lower end and $670 \mathrm{~nm}$ on the upper end.

Although we selected commercially available dichroic beamsplitters and emission filters in this work, it is possible to increase the collection efficiency of the conventional fluorescence channel by using custom dichroic beamsplitters with a transition edge closer to the excitation wavelength. For dual-objective systems, collecting salvaged fluorescence at both objectives can further improve the performance. Additionally, the detected salvaged 
fluorescence can be used to improve the localization precision but at the cost of image registration error and added complexity in the data analysis.

\section{Statistics}

The average separation between GM130 and GRASP65 (Fig. 3i) was determined to be not significantly different from zero $(P=0.62)$ by using a two-tailed Student's t-Test in

MATLAB.

\section{Reporting Summary}

Further information on research design is available in the Life Sciences Reporting Summary linked to this article.

\section{Data availability}

The datasets generated and/or analyzed during the current study, technical drawings and parts lists are available from the corresponding author upon request.

\section{Code availability}

Custom MATLAB code about the color assignment for the salvaged fluorescence approach used during the current study is available at: https://github.com/bewersdorflab/salvagedfluorescence.

\section{Supplementary Material}

Refer to Web version on PubMed Central for supplementary material.

\section{Acknowledgments}

We would like to thank R. Benavente (University of Wuerzburg) for sharing the anti-SYCP1-N antibody, A.E.S. Barentine and A.M. Ernst (Yale University) for comments on the manuscript, and C.G. Ebeling (Bruker Corp.) for help with the Vutara SRX software. This work was primarily supported by grants from the Wellcome Trust (203285/B/16/Z), the G. Harold and Leila Y. Mathers Foundation, and NIH (R01 GM118486, P30 DK045735 and NS36251). Y.L. acknowledges support from the EMBL Interdisciplinary Postdoc Programme (EIPOD) under Marie Curie Actions COFUND. J.B.G. and L.D.L. were funded by the Howard Hughes Medical Institute (HHMI).

\section{References}

1. Ladinsky MS, Mastronarde DN, McIntosh JR, Howell KE \& Staehelin LA Golgi structure in three dimensions: functional insights from the normal rat kidney cell. J. Cell Biol. 144, 1135-1149 (1999). [PubMed: 10087259]

2. Schroeder LK et al. Dynamic nanoscale morphology of the ER surveyed by STED microscopy. J. Cell Biol. 218, 83-96 (2019). [PubMed: 30442642]

3. Baddeley D \& Bewersdorf J Biological Insight from Super-Resolution Microscopy: What We Can Learn from Localization-Based Images. Annu. Rev. Biochem. 87, 965-989 (2018). [PubMed: 29272143]

4. Sigal YM, Zhou R \& Zhuang X Visualizing and discovering cellular structures with super-resolution microscopy. Science 361, 880-887 (2018). [PubMed: 30166485]

5. von Middendorff C, Egner A, Geisler C, Hell SW \& Schonle A Isotropic 3D Nanoscopy based on single emitter switching. Opt. Express 16, 20774-20788 (2008). [PubMed: 19065216]

6. Shtengel $\mathrm{G}$ et al. Interferometric fluorescent super-resolution microscopy resolves 3D cellular ultrastructure. Proc. Natl. Acad. Sci. U. S. A 106, 3125-3130 (2009). [PubMed: 19202073] 
7. Aquino D et al. Two-color nanoscopy of three-dimensional volumes by 4Pi detection of stochastically switched fluorophores. Nat. Methods 8, 353-359 (2011). [PubMed: 21399636]

8. Huang F et al. Ultra-High Resolution 3D Imaging of Whole Cells. Cell 166, 1028-1040 (2016). [PubMed: 27397506]

9. Van Engelenburg SB et al. Distribution of ESCRT machinery at HIV assembly sites reveals virus scaffolding of ESCRT subunits. Science 343, 653-656 (2014). [PubMed: 24436186]

10. Buttler CA et al. Single molecule fate of HIV-1 envelope reveals late-stage viral lattice incorporation. Nat. Commun. 9, 1861 (2018). [PubMed: 29748537]

11. Carlini L, Holden SJ, Douglass KM \& Manley S Correction of a Depth-Dependent Lateral Distortion in 3D Super-Resolution Imaging. PLoS ONE 10, e0142949 (2015). [PubMed: 26600467]

12. Li YM, Wu YL, Hoess P, Mund M \& Ries J Depth-dependent PSF calibration and aberration correction for 3D single-molecule localization. Biomedical Optics Express 10, 2708-2718 (2019). [PubMed: 31259045]

13. Burke D, Patton B, Huang F, Bewersdorf J \& Booth MJ Adaptive optics correction of specimeninduced aberrations in single-molecule switching microscopy. Optica 2, 177-185 (2015).

14. Schonle A \& Hell SW Fluorescence nanoscopy goes multicolor. Nat. Biotechnol. 25, 1234-1235 (2007). [PubMed: 17989684]

15. Bossi M et al. Multicolor far-field fluorescence nanoscopy through isolated detection of distinct molecular species. Nano Lett. 8, 2463-2468 (2008). [PubMed: 18642961]

16. Baddeley D et al. 4D super-resolution microscopy with conventional fluorophores and single wavelength excitation in optically thick cells and tissues. PLoS ONE 6, e20645 (2011). [PubMed: 21655189]

17. Lampe A, Haucke V, Sigrist SJ, Heilemann M \& Schmoranzer J Multi-colour direct STORM with red emitting carbocyanines. Biol. Cell. 104, 229-237 (2012). [PubMed: 22187967]

18. Zhang Z, Kenny SJ, Hauser M, Li W \& Xu K Ultrahigh-throughput single-molecule spectroscopy and spectrally resolved super-resolution microscopy. Nat. Methods 12, 935-938 (2015). [PubMed: 26280329]

19. Mlodzianoski MJ, Curthoys NM, Gunewardene MS, Carter S \& Hess ST Super-Resolution Imaging of Molecular Emission Spectra and Single Molecule Spectral Fluctuations. PLoS ONE 11, e0147506 (2016). [PubMed: 27002724]

20. Dempsey GT, Vaughan JC, Chen KH, Bates M \& Zhuang X Evaluation of fluorophores for optimal performance in localization-based super-resolution imaging. Nat. Methods 8, 1027-1036 (2011). [PubMed: 22056676]

21. Nieuwenhuizen RP et al. Measuring image resolution in optical nanoscopy. Nat. Methods 10, 557562 (2013). [PubMed: 23624665]

22. Ries J, Kaplan C, Platonova E, Eghlidi H \& Ewers H A simple, versatile method for GFP-based super-resolution microscopy via nanobodies. Nat. Methods 9, 582-584 (2012). [PubMed: 22543348]

23. Szymborska A et al. Nuclear Pore Scaffold Structure Analyzed by Super-Resolution Microscopy and Particle Averaging. Science 341, 655-658 (2013). [PubMed: 23845946]

24. Schucker K, Holm T, Franke C, Sauer M \& Benavente R Elucidation of synaptonemal complex organization by super-resolution imaging with isotropic resolution. Proc. Natl. Acad. Sci. U. S. A. 112, 2029-2033 (2015). [PubMed: 25646409]

25. Schmitt $\mathbf{J}$ et al. Transmembrane protein Sun2 is involved in tethering mammalian meiotic telomeres to the nuclear envelope. Proc. Natl. Acad. Sci. U. S. A 104, 7426-7431 (2007). [PubMed: 17452644]

26. Barr FA, Puype M, Vandekerckhove J \& Warren G GRASP65, a protein involved in the stacking of Golgi cisternae. Cell 91, 253-262 (1997). [PubMed: 9346242]

27. Fernandez-Busnadiego R, Saheki Y \& De Camilli P Three-dimensional architecture of extended synaptotagmin-mediated endoplasmic reticulum-plasma membrane contact sites. Proc. Natl. Acad. Sci. U. S. A. 112, E2004-2013 (2015). [PubMed: 25787254]

28. Chung J et al. PI4P/phosphatidylserine countertransport at ORP5- and ORP8-mediated ER-plasma membrane contacts. Science 349, 428-432 (2015). [PubMed: 26206935] 
29. Giordano $\mathrm{F}$ et al. $\mathrm{PI}(4,5) \mathrm{P}(2)$-dependent and $\mathrm{Ca}(2+)$-regulated ER-PM interactions mediated by the extended synaptotagmins. Cell 153, 1494-1509 (2013). [PubMed: 23791178]

30. Li X et al. Electron counting and beam-induced motion correction enable near-atomic-resolution single-particle cryo-EM. Nat. Methods 10, 584-590 (2013). [PubMed: 23644547]

31. Wang $Y$ et al. Localization events-based sample drift correction for localization microscopy with redundant cross-correlation algorithm. Opt. Express 22, 15982-15991 (2014). [PubMed: 24977854]

32. Los GV et al. HaloTag: a novel protein labeling technology for cell imaging and protein analysis. ACS Chem. Biol. 3, 373-382 (2008). [PubMed: 18533659]

33. Keppler A et al. A general method for the covalent labeling of fusion proteins with small molecules in vivo. Nat. Biotechnol. 21, 86-89 (2003). [PubMed: 12469133]

34. Takakura $\mathrm{H}$ et al. Long time-lapse nanoscopy with spontaneously blinking membrane probes. Nat. Biotechnol. 35, 773-780 (2017). [PubMed: 28671662]

35. Douglass KM, Sieben C, Archetti A, Lambert A \& Manley S Super-resolution imaging of multiple cells by optimised flat-field epi-illumination. Nat Photonics 10, 705-708 (2016). [PubMed: 27818707]

36. Deschamps J, Rowald A \& Ries J Efficient homogeneous illumination and optical sectioning for quantitative single-molecule localization microscopy. Opt. Express 24, 28080-28090 (2016). [PubMed: 27906373]

37. Pallikkuth $S$ et al. Sequential super-resolution imaging using DNA strand displacement. PLoS ONE 13, e0203291 (2018). [PubMed: 30169528]

38. Jungmann R et al. Multiplexed 3D cellular super-resolution imaging with DNA-PAINT and Exchange-PAINT. Nat. Methods 11, 313-318 (2014). [PubMed: 24487583]

\section{References}

39. Nemoto Y \& De Camilli P Recruitment of an alternatively spliced form of synaptojanin 2 to mitochondria by the interaction with the PDZ domain of a mitochondrial outer membrane protein. EMBO J. 18, 2991-3006 (1999). [PubMed: 10357812]

40. Huang F et al. Video-rate nanoscopy using sCMOS camera-specific single-molecule localization algorithms. Nat. Methods 10, 653-658 (2013). [PubMed: 23708387]

41. Hanser BM, Gustafsson MG, Agard DA \& Sedat JW Phase-retrieved pupil functions in wide-field fluorescence microscopy. J. Microsc. 216, 32-48 (2004). [PubMed: 15369481]

42. Hoess P, Mund M, Reitberger M \& Ries J Dual-Color and 3D Super-Resolution Microscopy of Multi-protein Assemblies. Methods Mol. Biol. 1764, 237-251 (2018). [PubMed: 29605918]

43. Huang F, Schwartz SL, Byars JM \& Lidke KA Simultaneous multiple-emitter fitting for single molecule super-resolution imaging. Biomedical Optics Express 2, 1377-1393 (2011). [PubMed: 21559149] 


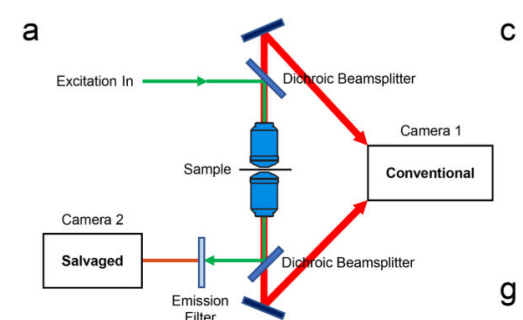

$\mathrm{b}$

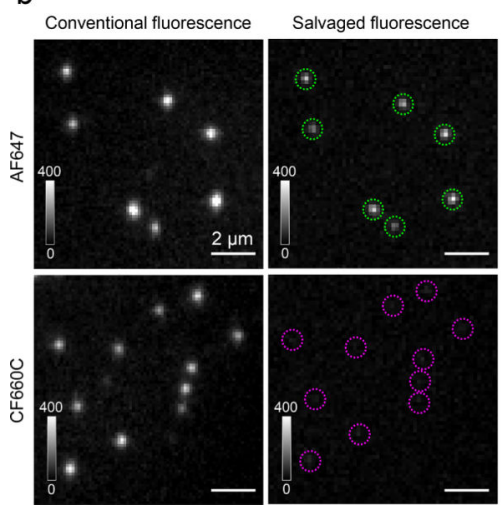

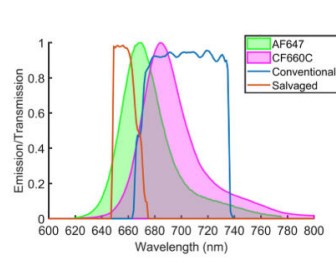

g

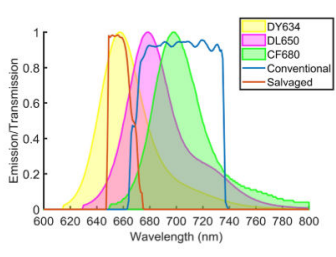

d

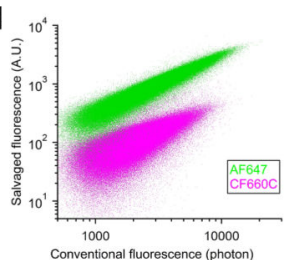

$\mathrm{h}$
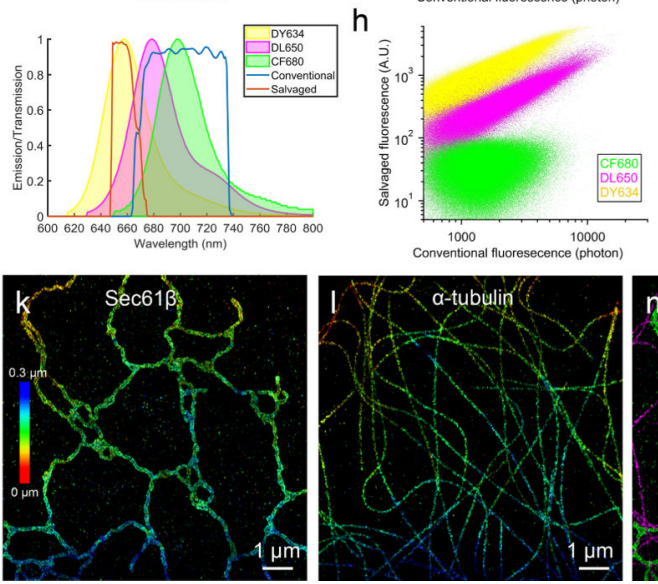

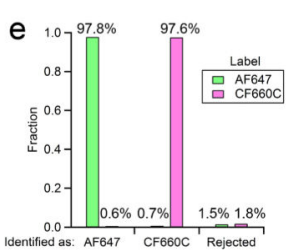

i
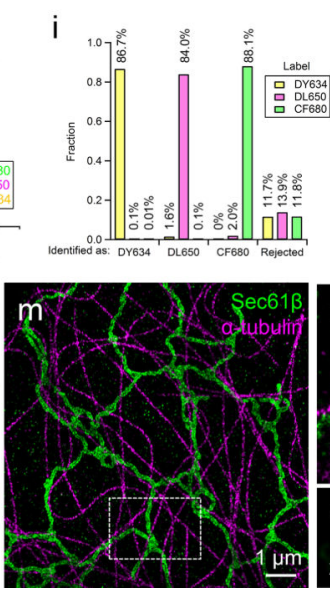

f
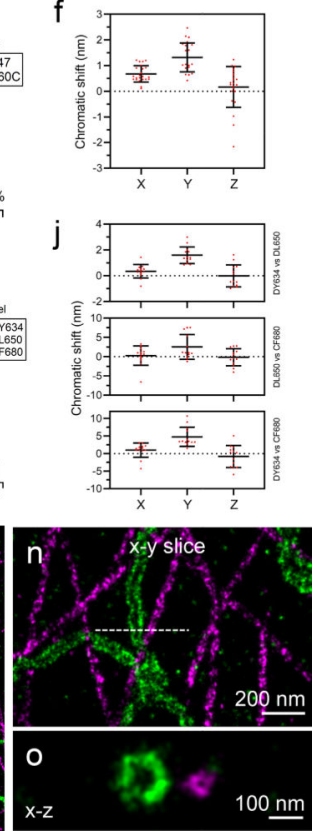

Figure 1: Characterization of multicolor 4Pi-SMS imaging using salvaged fluorescence. a, Schematic of the multicolor 4Pi-SMS microscope. Camera 1 captures conventional fluorescence. Camera 2 captures salvaged fluorescence. b, Conventional and salvaged fluorescence images of single molecules of AF647 and CF660C (Supplementary Video 1).

Dashed circles indicate the positions of single molecules observed in the conventional fluorescence channel. c, Emission spectra of AF647 and CF660C and transmission profiles for conventional and salvaged fluorescence. $\mathbf{d}$, Scatter plot of salvaged fluorescence versus conventional fluorescence intensities of localized dye molecules in two single-color microtubule samples (shown in Supplementary Fig. 4) on a logarithmic scale. e, Cross-talk and rejected fraction for the two dyes. f, Chromatic shift between AF647 and CF660C in each dimension, determined from 23 images (each $20 \times 20 \mu \mathrm{m}$ ). g, Emission spectra of DY634, DL650 and CF680 and the transmission profiles for conventional and salvaged fluorescence. $\mathbf{h}$, Scatter plot of salvaged fluorescence versus conventional fluorescence intensities of localized dye molecules in three single-color microtubule samples (shown in Supplementary Fig. 5) on a logarithmic scale. i, Cross-talk and rejected fraction for the three dyes. j, Chromatic shift between each dye pair, determined from 13 images (each $20 \times 20$ $\mu \mathrm{m})$. k-m, Two-color images of microtubules and ER membrane in a COS-7 cell. $\mathbf{n}, 50-\mathrm{nm}$ thick x-y slice of the boxed region in (m). o, 20-nm thick x-z cross-section along the dashed line in $(\mathbf{n})$. Data are presented as mean \pm s.d. in $(\mathbf{f}, \mathbf{j})$. Representative images of two (b) or five (k-o) independent experiments are shown. Spectra shown in $(\mathbf{c}, \mathbf{g})$ were obtained from the respective manufacturers' websites. 

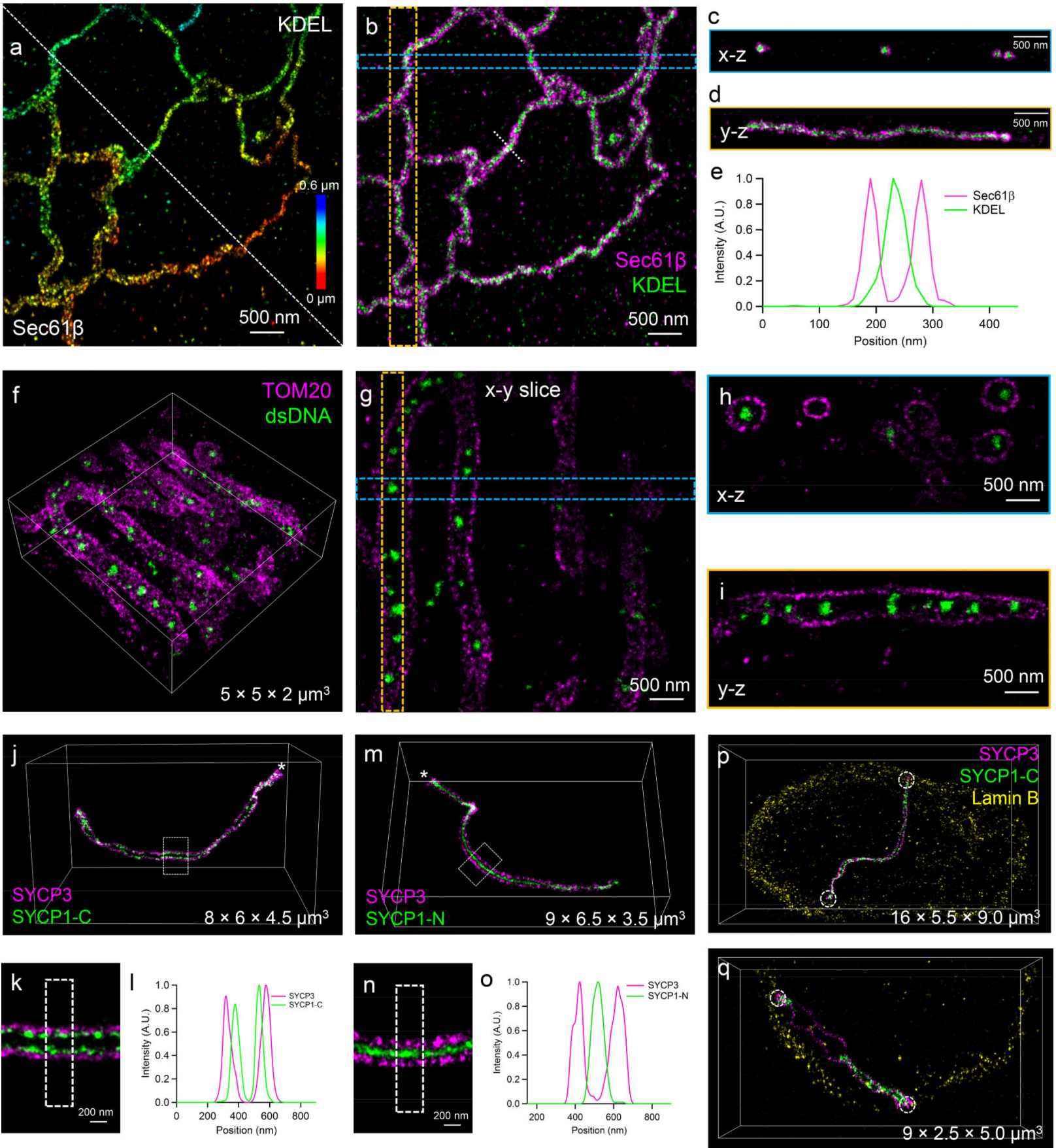

Figure 2: Multicolor 4Pi-SMS images of ER, mitochondria, and synaptonemal complexes. $\mathbf{a}, \mathbf{b}$, Two-color image of ER membrane and lumen in a COS-7 cell (Supplementary Video 2). The lower left and upper right corners show the Sec61 $\beta$ labeling and KDEL labeling, respectively. Rainbow color denotes depth in (a). Overlay image in (b). c, X-Z view of the blue dashed box (200 nm wide) in (b). d, y-z view of the orange dashed box (400 nm wide) in (b). e, Intensity profile along the white dashed line in (b). f, Two-color image of outer mitochondrial membrane and mitochondrial dsDNA in a HeLa cell (Supplementary Video 3). g, A 200-nm thick $x-y$ slice of the image in (f). h, $x-z$ view of the blue dashed box (300 
$\mathrm{nm}$ wide) in (g). $\mathbf{i}, \mathrm{y}-\mathrm{z}$ view of the orange dashed box (300 $\mathrm{nm}$ wide) in (g). $\mathbf{j}$,

Computationally isolated two-color image of SYCP3 and SYCP1-C in a synaptonemal complex (Supplementary Fig. 8a,b and Supplementary Video 4, part I). k, Magnified image of the boxed region in (j). i, Intensity profile along the dashed boxed region in $(\mathbf{k})$. $\mathbf{m}$, Computationally isolated two-color image of SYCP3 and SYCP1-N in a synaptonemal complex (Supplementary Fig. 8c,d and Supplementary Video 4, part II). n, Magnified image of the boxed region in $(\mathbf{m})$. o, Intensity profile along the dashed boxed region in (n). p,q, Three-color images of SYCP3 and SYCP1-C on two computationally isolated synaptonemal complexes next to Lamin B (Supplementary Fig. 8e,f and Supplementary Video 4, part III). White dashed circles indicate where synaptonemal complexes contact the nuclear lamina.

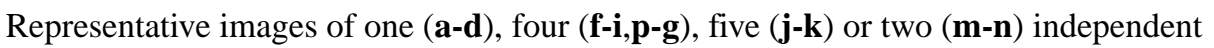
experiments are shown. 

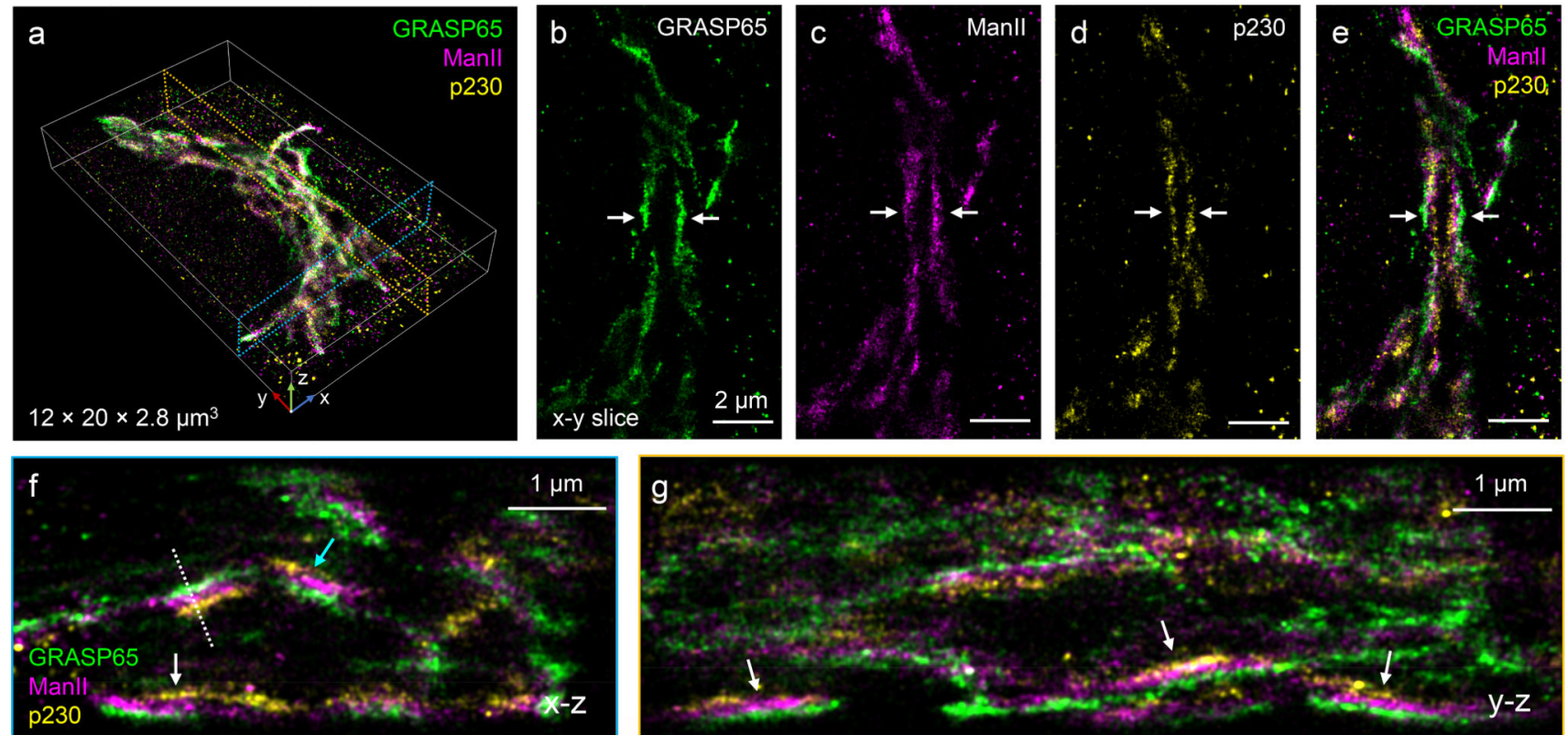

h
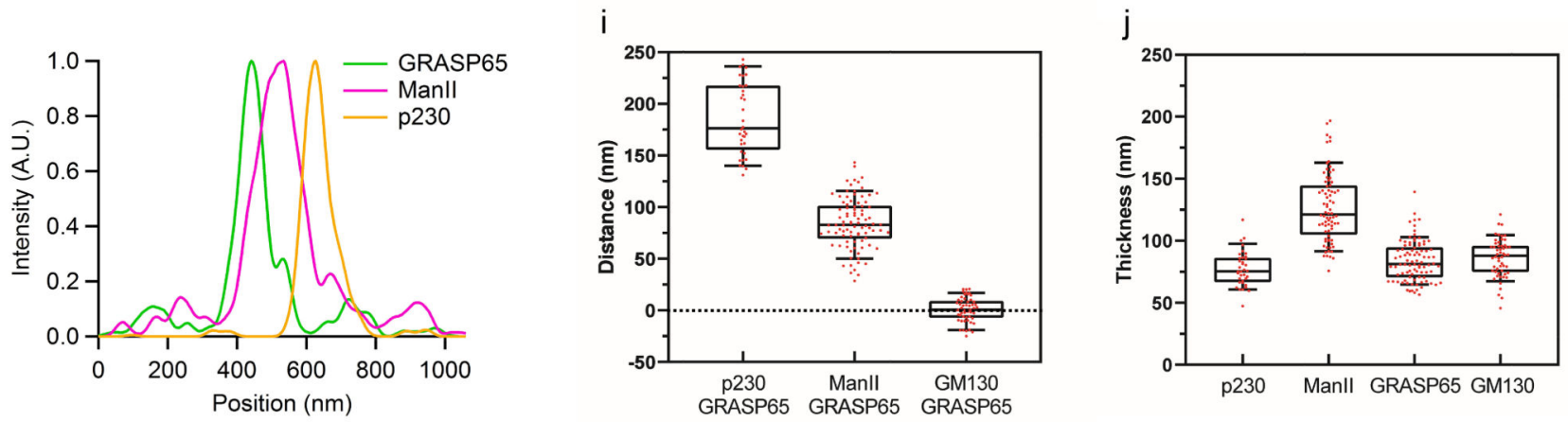

Figure 3: Complex stacked architecture of the Golgi apparatus.

a, 3D overlay image of cis (GRASP65), medial (ManII), and trans (p230) Golgi proteins in a HeLa cell (Supplementary Fig. 9 and Supplementary Video 5, part I). b-e, 500-nm thick x-y slice of data shown in (a). f, 1- $\mu \mathrm{m}$ thick $x-z$ cross-section centered at the blue dashed region in (a). $\mathbf{g}, 1-\mu \mathrm{m}$ thick $\mathrm{y}-\mathrm{z}$ cross-section centered at the orange dashed region in (a). $\mathbf{h}$, Intensity profile along the dashed line in (f). (i) Distance between the peak intensities of each pair of Golgi proteins (p230-GRASP65, $n=36$ from 9 cells; ManII-GRASP65, $n=90$ from 18 cells; GM130-GRASP65, $\mathrm{n}=54$ from 9 cells). $\mathbf{j}$, Thickness of Golgi regions, measured as the full-width at half-maximum of the intensity profile (p230, $\mathrm{n}=36$ from 9 cells; ManII, $\mathrm{n}=76$ from 18 cells; GRASP65, $\mathrm{n}=90$ from 18 cells; GM130, $\mathrm{n}=54$ from 9 cells). Arrows denote where the cis-medial-trans stack is visible (b-e,f,g). Median and interquartile range are shown with whiskers drawn down to the 10th percentile and up to the 90th percentile $(\mathbf{i}, \mathbf{j})$. Representative images of three independent experiments are shown (ag). 


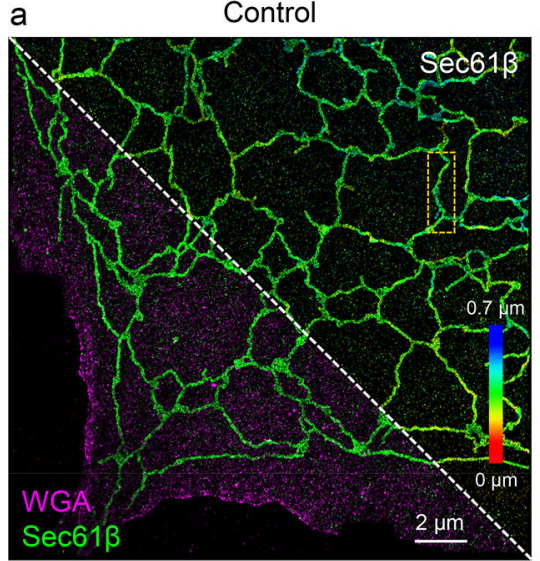

C

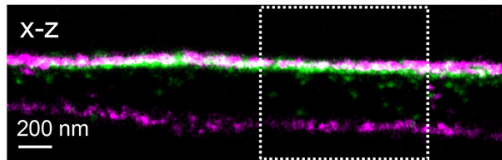

d

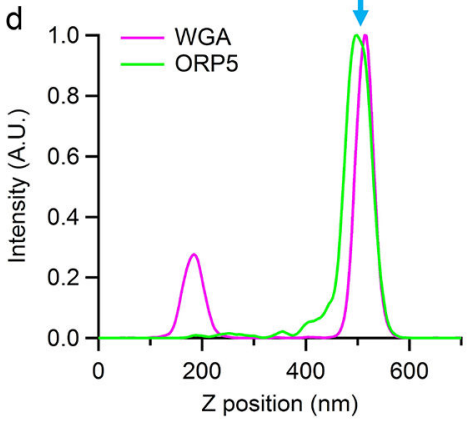

$1 \sqrt{1}$

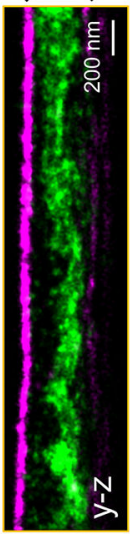

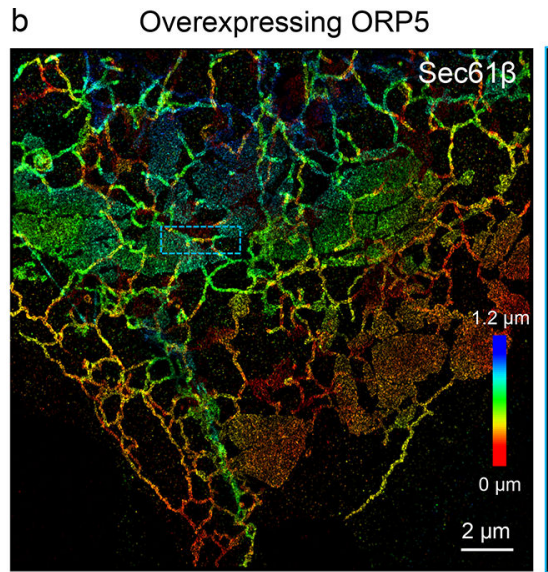

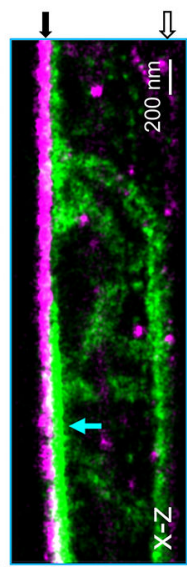

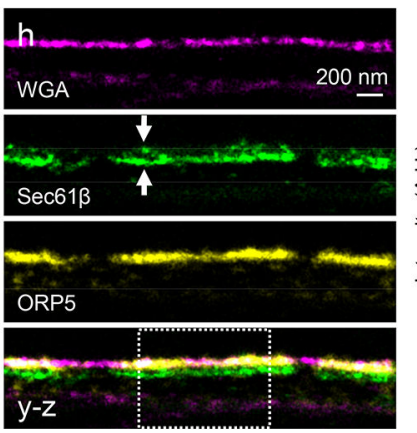

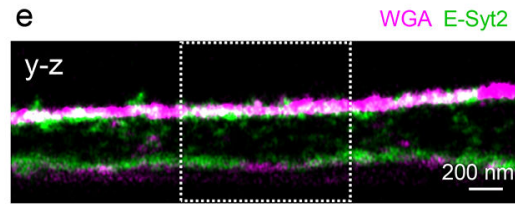

g
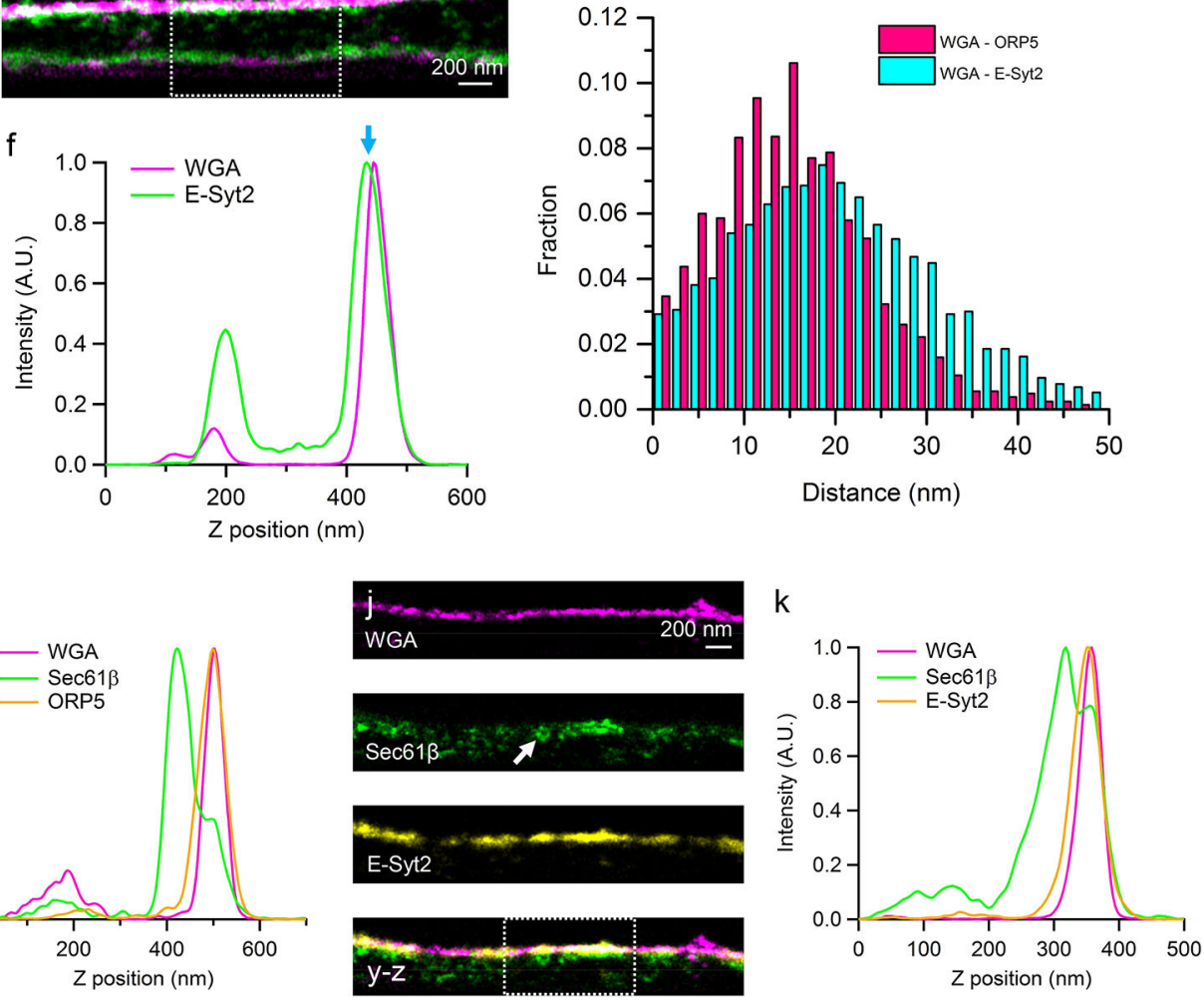

Figure 4: The 3D architecture of ER-PM contact sites.

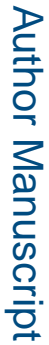

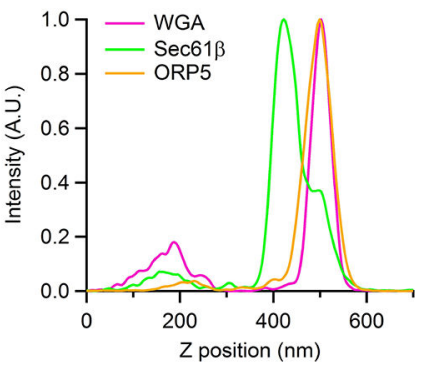

a, Two-color image of ER (Sec61 $\beta$ ) and PM (WGA) in a COS-7 cell (Supplementary Video 6 , part I). Top right half shows the ER where rainbow color denotes depth. Bottom left half overlays ER and PM. Right panel shows magnified y-z view of boxed region. b, Two-color image of ER and PM in a cell overexpressing mCherry-ORP5 (Supplementary Video 6, part II). Right panel shows magnified $x-z$ view of boxed region. Solid and outlined arrows in (a,b) point to the top and bottom PM, respectively. c, Two-color ORP5 and PM X-Z view, overview shown in (Supplementary Fig. 11b). d, Axial intensity profile across the dashed box in (c). e, Two-color E-Syt2 and PM y-z view, overview shown in (Supplementary Fig. 
11c). f, Axial intensity profile across the dashed box in (e). Blue arrows in (d,f) indicate the distance between PM and contact site proteins. g, Histograms of the distance between PM and contact site proteins (from $>2800$ subregions of $100 \times 100 \mathrm{~nm}$ size, $\mathrm{n}=4$ cells per condition) (See Methods). h, Three-color ER, ORP5 and PM y-z view, overview shown in (Supplementary Fig. 12g and Supplementary Video 7, part I). White arrows indicate the top and bottom membranes of the ER. i, Axial intensity profile across the dashed box in (h). $\mathbf{j}$, Three-color ER, E-Syt2, and PM y-z view, overview shown in (Supplementary Fig. 12h and Supplementary Video 7, part II). White arrow points to an ER tubule. k, Axial intensity profile across the dashed box in (j). Representative images of four (a), three (b-c) or two $(\mathbf{e}, \mathbf{h}, \mathbf{j})$ independent experiments are shown. 